\title{
THE CAVE OF THEOPETRA, KALAMBAKA: RADIOCARBON EVIDENCE FOR 50,000 YEARS OF HUMAN PRESENCE
}

\author{
Yorgos Facorellis $^{1,2} \bullet$ Nina Kyparissi-Apostolika ${ }^{3} \bullet$ Yannis Maniatis $^{1}$
}

\begin{abstract}
The cave of Theopetra is located on the northeast side of a limestone rock formation, $3 \mathrm{~km}$ south of Kalambaka $\left(21^{\circ} 40^{\prime} 46^{\prime \prime} \mathrm{E}, 39^{\circ} 40^{\prime} 51^{\prime \prime} \mathrm{N}\right)$, in Thessaly, central Greece. It is a unique prehistoric site for Greece, as the Middle and Upper Paleolithic, Mesolithic, and Neolithic periods are present here, bridging the Pleistocene with the Holocene. Several alternations of the climate during the Pleistocene are recognized in its stratigraphy. Among the most striking finds, two human skeletons, one from the Upper Paleolithic period after the Last Glacial Maximum and one from the Mesolithic period, should be emphasized, while in a deep Middle Paleolithic layer, the oldest human footprints, with remains of fire, were uncovered.

During the 13 years of excavation, evidence of human activity suitable for radiocarbon dating was collected, such as charcoal samples from hearths and bones from the two human skeletons. The use of proportional counters for the measurement of ${ }^{14} \mathrm{C}$ in combination with the recent improvement of the calibration curve has enabled the production of high-precision reliable ages. Sixty ${ }^{14} \mathrm{C}$-dated samples, originating from 19 pits and from depths ranging from $0.10 \mathrm{~m}$ to $4.20 \mathrm{~m}$, have already provided an absolute time framework for the use of the cave. The earliest limit of human presence probably exceeds $48,000 \mathrm{BP}$ and the latest reaches World War II. Within these limits the ${ }^{14} \mathrm{C}$ dating of samples from consecutive layers, in combination with the archaeological data, permits the resolution of successive anthropogenic and environmental events.
\end{abstract}

\section{INTRODUCTION}

Paleolithic research in Thessaly started 35 years ago by the German Archaeological School under Vladimir Milojcic in the area of the river Peneios. Flintstone tools from the Middle Paleolithic period, as well as fossil bones from the same period were found in that area and were dated from around 44,000 BP (Milojcic et al. 1965). However, excavations have been made in Thessaly in Neolithic locations from the beginning of the 20th century, and despite the very poor means used, they managed to promote Thessaly as the bastion of the Neolithic civilization in Greece (Tsountas 1908).

In 1987, C Runnels from Boston University started surface research with the goal to determine chronologically the Middle Paleolithic period in Greece. He found and collected tools from the Middle and Upper Paleolithic period without finding, however, any workshops for their production (Runnels 1988, 1993). Thus, he was led to the conclusion that during this period the area of Thessaly was not inhabited but was only periodically visited by various groups of hunters from other areas, and that the tools found from the Upper Paleolithic period should belong to them. He concluded also that the caves of Thessaly should not have been inhabited during the Paleolithic period. Both he and C Perlès (1988), with the evidence at hand, concluded that the Mesolithic phase was not present in Thessaly. They suggested that in Franchthi cave the Mesolithic groups evolved to Neolithic, in Thessaly the Neolithic settlements were founded by groups foreign to the area but not necessarily to the Helladic world. D Theocharis (1970), who was the first to visit the cave of Theopetra, announced that he considers it to be one of the locations in Greece with findings from the Middle Paleolithic period.

In 1987 excavations began in Theopetra under the direction of N Kyparissi-Apostolika, which were meant to give some answers to the mystery of Paleolithic Thessaly. The cave is located on the northeast side of a limestone rock formation (Figure 1) a hill $3 \mathrm{~km}$ south of the town of Kalambaka in Thessaly, central Greece $\left(21^{\circ} 40^{\prime} 46^{\prime \prime} \mathrm{E}, 39^{\circ} 40^{\prime} 51^{\prime \prime} \mathrm{N}\right)$. The formation of the limestone rock has been dated to the Upper Cretaceous period, 135-65 million years BP (Ardaens 1978; Karkanas 1999).

${ }^{1}$ Laboratory of Archaeometry, N.C.S.R. "Demokritos", 15310 Ag. Paraskevi, Greece

${ }^{2}$ Corresponding author. Email: yfacorellis@ims.demokritos.gr.

${ }^{3}$ Ephorate of Paleoanthropology and Speleology, Ardittou 34B, 11636 Athens, Greece

(C) 2001 by the Arizona Board of Regents on behalf of the University of Arizona

RADIOCARBON, Vol 43, Nr 2B, 2001, p 1029-1048

Proceedings of the 17 th International ${ }^{14} \mathrm{C}$ Conference, edited by I Carmi and E Boaretto 


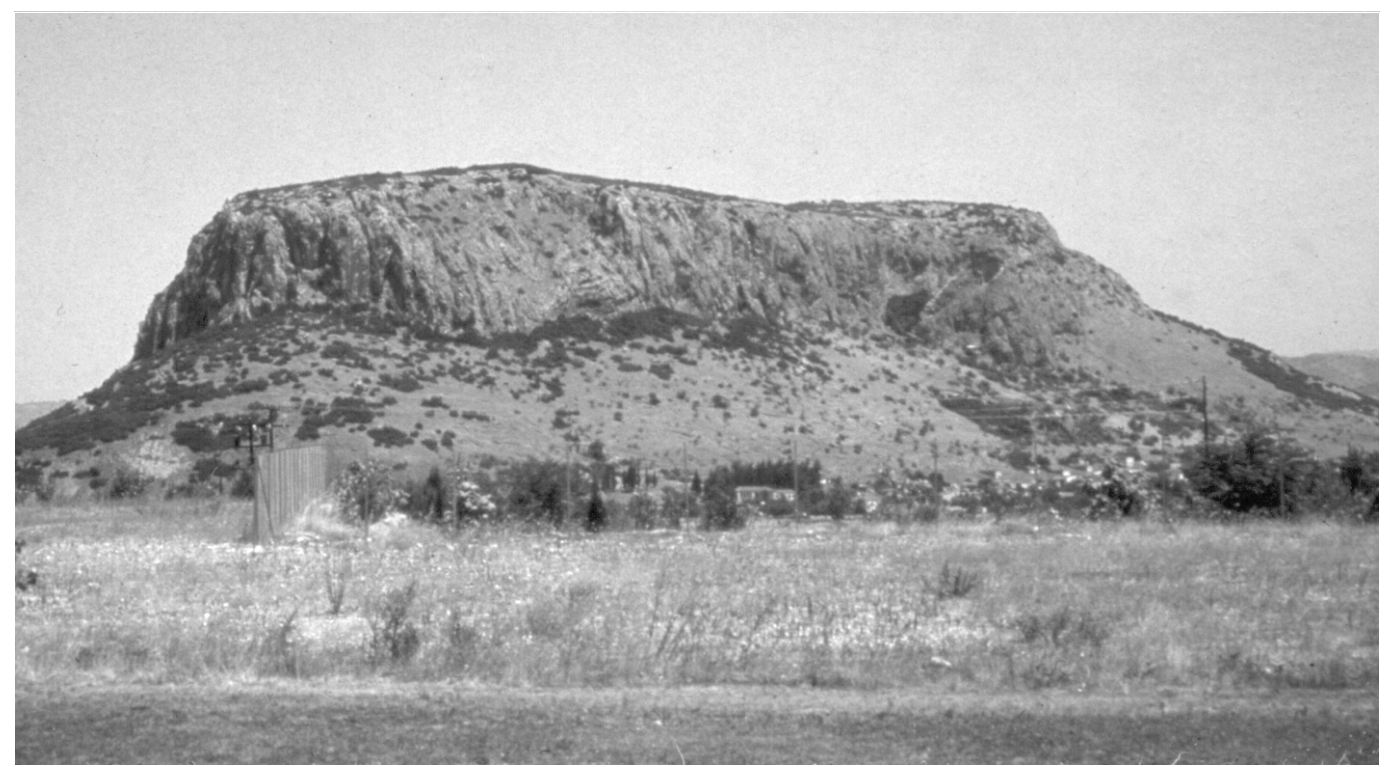

Figure 1 The rock formation of Theopetra. View from the southwest.

From the entrance of the cave, which is apsidal and large (approximately $17 \times 3 \mathrm{~m}$ ), almost as much as its inner width, one can see across to the rock formations of Meteora, which are famous for its Byzantine monasteries. The view is exactly the same as what the first inhabitants of the cave would have seen. A hundred meters below the cave, the river Lethaios, a tributary of Peneios, runs through the plain of Thessaly and becomes almost dry in the summer. The difference in height between the plain and the cave entrance is about $100 \mathrm{~m}$. The interior of the cave (Figure 2) is about $500 \mathrm{~m}^{2}$, almost quadrilateral in shape, with the exception of its periphery where small cavities are formed, which have the same signs of inhabitancy as the rest of the cave (Kyparissi-Apostolika 1990).

The target of this work is to provide an absolute chronological framework for the habitation sequence of the cave. Using detailed stratigraphic sampling and high-precision dating, we attempt to resolve phases and determine the time of major cultural changes. Also, we aim to answer questions about the existence of the Mesolithic and the Paleolithic periods in Thessaly.

\section{METHODS}

All the samples taken to the laboratory for ${ }^{14} \mathrm{C}$ dating were charcoal pieces, except for two samples of bones originating from two human skeletons and one soil sediment rich in charcoal particles. All samples were chemically pretreated to remove any carbon compounds of non-archaeological origin (Longin 1971; Olsson 1979; Mook and Streurman 1983; Brown et al. 1988; Hedges and Law 1989; Arslanov and Svezhentsev 1993; Facorellis 1996).

After the chemical pretreatment the samples are converted to $\mathrm{CO}_{2}$ using the de Vries continuous combustion technique (de Vries and Barendsen 1953; Facorellis 1996). Then the produced $\mathrm{CO}_{2}$ undergoes several purification steps, described elsewhere (Facorellis 1996), and finally it is measured inside cylindrical gas proportional counters (Facorellis et al. 1997). The laboratory possesses 8 such counters, four with a capacity of $4 \mathrm{~L}$ and four with $2.5 \mathrm{~L}$. 


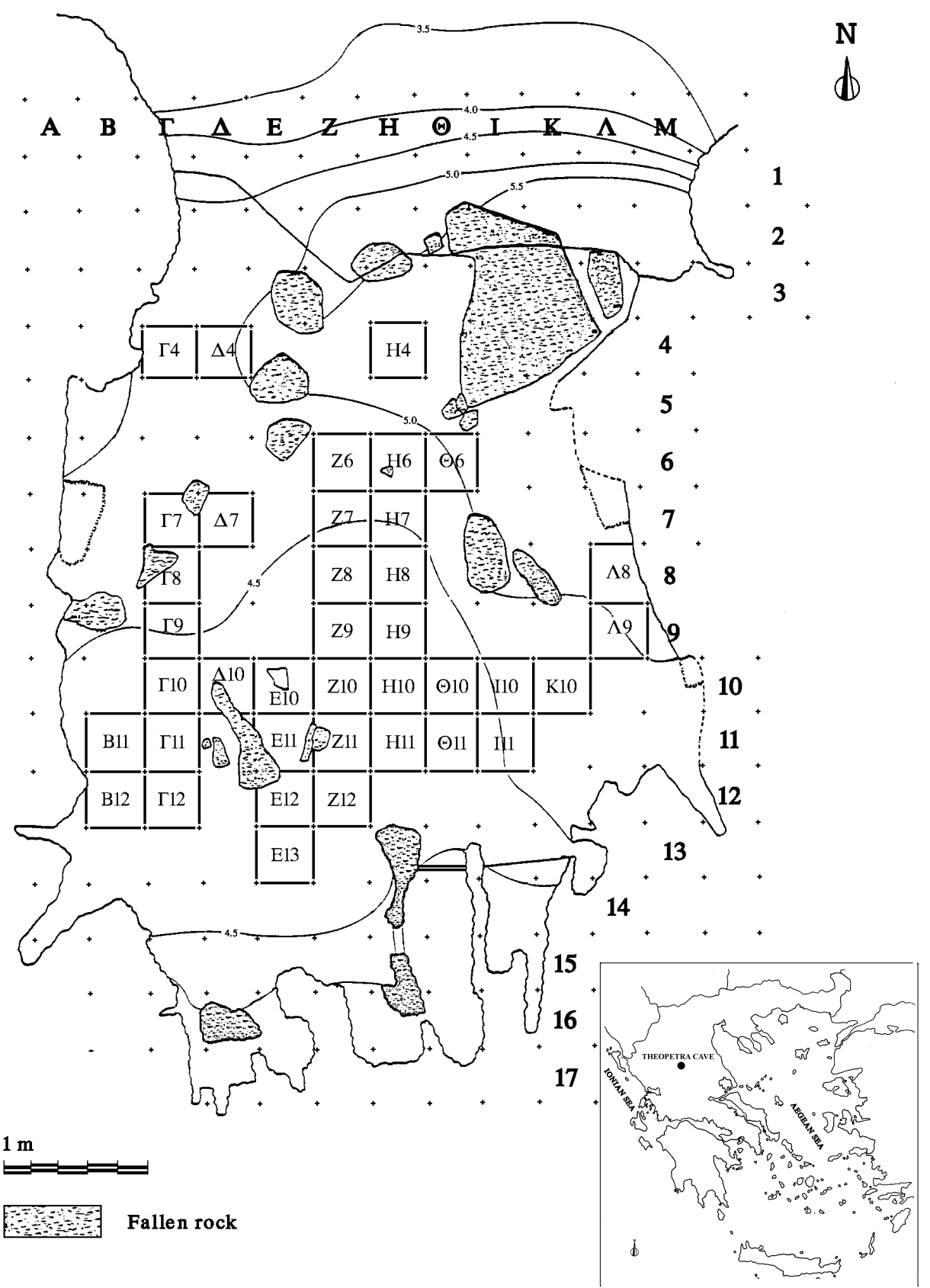

Figure 2 Plan of the Theopetra cave showing the excavated pits and map of Greece 
The smaller amount of ${ }^{14} \mathrm{C}$ that is possible to be detected by these counters corresponds to an age of about 50,000 BP (Facorellis and Maniatis 1999). This fact is particularly important, as it allowed the dating of samples originating from all the layers of the Theopetra cave, which cover all the age ranges of the ${ }^{14} \mathrm{C}$ dating method.

\section{RESULTS}

During the 13 years of archaeological research, 38 pits measuring $2 \times 2 \mathrm{~m}$ were excavated covering one third of the cave's surface. The pits were dug to a depth that fluctuated from $0.5 \mathrm{~m}$ to about $6 \mathrm{~m}$, which was the maximum thickness of the deposits in the center of the cave (trench Z6-Z7-Z8-Z9) (Figure 2).

Up to now, $60{ }^{14} \mathrm{C}$-dated samples, originating from 19 pits and from depths ranging from $0.10 \mathrm{~m}$ to $4.20 \mathrm{~m}$, have already given an absolute time framework for the use of the cave. The earliest limit of human presence probably exceeds 48,000 BP, and the latest reaches World War II.

Table 1 presents all the necessary information on the dated samples (laboratory code number, date of sampling, pit, depth, and type). Except for the above sample details, the conventional ${ }^{14} \mathrm{C}$ dates sorted by age are also given. For the age correction due to the isotopic fractionation the usual for the charcoal samples value $\delta^{13} \mathrm{C}=-25.00 \%$ was used (Stuiver and Polach 1977; Polach 1976). In the case of the human bone sample the expected value $\delta^{13} \mathrm{C}=-21.00 \%$ was used which corresponds to a human whose protein uptake is based on a Calvin-Benson (C3) metabolic type food chain (Johansen et al. 1986; Stuiver and Reimer 1993). Experience has shown that practically all bone samples excavated in Greece give the value of $\delta^{13} \mathrm{C}=-21.00 \%$ \pm 1 .

\section{Calibration}

The table also shows the calibrated ages expressed in cal BP, as well as the corresponding calendar dates of the samples within 1 and 2 standard deviations (probability $68.3 \%$ and $95.4 \%$, respectively). The calibration of the conventional ${ }^{14} \mathrm{C}$ ages was performed in three different ways depending on the time range as follows:

1. For those that fall into the time period between 24,000 cal BP until present they were calibrated with the new international calibration curve using the Calib 4.1.2 program of the Quaternary Isotope Laboratory of the University of Washington (Stuiver and Reimer 1993; Stuiver et al. 1998).

2. For those that fall into the time period from 40,000 to 24,000 cal BP (samples DEM-61, DEM223, DEM-374 and DEM-247) they were calibrated approximately using the 2nd order polynomial equation proposed by Bard et al. (1998). This equation can be applied to the conventional radiocarbon ages older than 10,000 BP (beyond the climatic episode Younger Dryas):

$$
[\mathrm{cal} \mathrm{BP}]=-30,126 \times 10^{-6} \times\left[\text { age }{ }^{14} \mathrm{C} \mathrm{BP}\right]^{2}+12,896 \times\left[\text { age }{ }^{14} \mathrm{C} \mathrm{BP}\right]-1005
$$

3. Finally, for those that fall into the time period 48,000-40,000 cal BP (samples DEM-134, DEM-74, DEM-133, DEM-140 and DEM-613) they were calibrated approximately using the magnetic calibration curve based on the ${ }^{14} \mathrm{C}$ production change versus the intensity of the geomagnetic field proposed by Laj et al. (1996), whose observations are confirmed by Voelker et al. (1998). It is worth noticing that according to this curve at around 46,000 BP the difference between the conventional ${ }^{14} \mathrm{C}$ age and the calibrated age appears to be almost zero. This fact means that the conventional ${ }^{14} \mathrm{C}$ age of the sample DEM-613 equals the calibrated one expressed in cal BP. However according to Kitagawa and van der Plicht (1998), who studied the 
fluctuation of the ${ }^{14} \mathrm{C}$ production in the atmosphere during the last 45,000 years based on systematic dating of the varves in the lake Suigetsu in Japan, it seems that at these age limits the conventional ${ }^{14} \mathrm{C}$ ages continue to be about 2000 years younger than the calibrated ones. Therefore, the calibrated ages of the samples from that time period shown in Table 1 can only be indicative for the present.

Figure 3 shows the probability distribution of the calibrated ${ }^{14} \mathrm{C}$ ages (cal BP) of all the samples from Theopetra cave sorted by age. The length of each bar represents the age range, and the height represents the percent probability that the sample is in the specific range (Maniatis and Kromer 1990). The shaded bars represent a tentative calibrated age as mentioned above.

\section{Dating the Cave Stratigraphy}

The deposits of Theopetra cave are the result of a sequence of recurrent natural and anthropogenic episodes. The natural deposits are due to the action of ground karstic waters covering occasionally the underlying anthropogenic layers, which are characterized by very thick ash remains (Karkanas 1999; Karkanas et al. 1999).

Although all the excavated layers of the cave with evidence for human activities (hearths) have been systematically sampled and dated so far, there are time intervals from which there are no ${ }^{14} \mathrm{C}$ dated samples, as can be seen in Table 1 and Figure 3. The major age gap begins from about 35,000 and ends at about 20,000 cal BP (DEM-223 and DEM-254). However, it is interrupted at around 30,000 cal BP by human presence (DEM-61). The micromorphological study of the sediments carried out by Karkanas and Weiner (2000) revealed the existence of several cold phases attributed to stadials of the last glacial. A major cold phase with temperatures below $0{ }^{\circ} \mathrm{C}$ is identified present in the sediments from 39,000 cal BP up to the end of the last glacial maximum at around 19,000 cal BP. This explains the absence of hearths and therefore human occupation in the cave at this period. This major cold phase is interrupted at 30,000 cal BP with milder conditions as evidenced by the sediment examination, and this explains again the presence of human occupation (sample DEM-61) (Figure 3). According to the same study, a cold episode is also present between 46,000 and 40,000 cal BP. This phase cannot be excluded from the ${ }^{14} \mathrm{C}$ dates but cannot be equally verified as the error bars are large and the age gaps cannot show up clearly.

A minor age gap between around 13,000 cal BP (DEM-249) and 11,200 cal BP (DEM-142) recorded in our data (Figure 3) may be due to a weaker and brief cold episode attributed to the Younger Dryas climatic event (Karkanas and Weiner 2000).

Another age gap spanning from $6300 \mathrm{cal}$ BP (DEM-141) ending at around $1200 \mathrm{cal}$ BP (DEM-225) is most probably due to repeated flooding by ground water originating from the karstic aquifers at the southern part of the cave, which eroded the uppermost anthropogenic deposits. The existence of a paleosurface higher than the actual surface of the cave is evidenced in the form of a relatively large band of brecciated layers adhering to the cave wall and containing the remains of anthropogenic deposits and Neolithic sherds. This band continues around the cave wall $2 \mathrm{~m}$ above the present surface at the back, and 1-1.5 m near the entrance. The water action must have caused the disturbance of the layers in certain areas of the cave. This is testified by the presence of the sample DEM-250 $(4542 \pm 240 \mathrm{cal} \mathrm{BP})$ at $2.77 \mathrm{~m}$ depth originating from an infill of a void, while the corresponding layers at these depths give ages over $15,000 \mathrm{cal} \mathrm{BP}$. This is further supported by the age $(6290 \pm 111 \mathrm{cal}$ BP) of the sample DEM-141, which comes from inside a heap of deposits, cemented in a stalagmite standing about $1 \mathrm{~m}$ above the present cave surface. These heaps, found at different points of the cave, consist of remains of Neolithic deposits cemented in situ by stalagmitic material due to intense water 
Table 1 Summary of radiocarbon dating results of samples from the Cave of Theopetra

\begin{tabular}{|c|c|c|c|c|c|c|c|c|c|}
\hline Lab code & $\begin{array}{l}\text { Date of } \\
\text { sampling } \\
(\mathrm{d} / \mathrm{m} / \mathrm{yr})\end{array}$ & Pit & Depth & Type & $\begin{array}{l}\delta^{13} \mathrm{C} \\
(\% o)\end{array}$ & Age (BP) & $\begin{array}{l}\text { Calibrated age } \\
\quad \text { (cal BP) }\end{array}$ & Calendar age & Probability \\
\hline DEM-244 & $19 / 8 / 1991$ & $\mathrm{Z} 12$, Northeastern region & $1.81 \mathrm{~m}$ & Charcoal & -25.00 & $118 \pm 76$ & $\begin{array}{l}270-15 \\
290-4\end{array}$ & $\begin{array}{l}1680-1940 \mathrm{AD} \\
1670-1955 \mathrm{AD}\end{array}$ & $\begin{array}{l}68.3 \% \\
95.4 \%\end{array}$ \\
\hline DEM-75 & 7/7/1989 & $\mathrm{Z} 10$ & $1.44 \mathrm{~m}$ & Charcoal & -25.00 & $201 \pm 45$ & $\begin{array}{l}297-3 \\
419-0\end{array}$ & $\begin{array}{l}1653-1955 \mathrm{AD} \\
1530-1955 \mathrm{AD}\end{array}$ & $\begin{array}{l}68.3 \% \\
95.4 \%\end{array}$ \\
\hline DEM-76 & $7 / 1989$ & $\mathrm{Z} 10$ & $1.97 \mathrm{~m}$ & Charcoal & -25.00 & $273 \pm 210$ & $\begin{array}{l}500-5 \\
630-1\end{array}$ & $\begin{array}{l}1450-1955 \mathrm{AD} \\
1330-1955 \mathrm{AD}\end{array}$ & $\begin{array}{l}68.3 \% \\
95.4 \%\end{array}$ \\
\hline DEM-60 & $14 / 4 / 1988$ & Disturbed layer & & Charcoal & -25.00 & $383 \pm 48$ & $\begin{array}{l}503-330 \\
513-314\end{array}$ & $\begin{array}{l}1450-1620 \mathrm{AD} \\
1440-1640 \mathrm{AD}\end{array}$ & $\begin{array}{l}68.3 \% \\
95.4 \%\end{array}$ \\
\hline DEM-243 & 8/8/1991 & Z12, Southwestern region & $1.01-1.21 \mathrm{~m}$ & Charcoal & -25.00 & $607 \pm 150$ & $\begin{array}{l}710-500 \\
890-310\end{array}$ & $\begin{array}{l}1250-1460 \mathrm{AD} \\
1060-1640 \mathrm{AD}\end{array}$ & $\begin{array}{l}68.3 \% \\
95.4 \%\end{array}$ \\
\hline DEM-124 & $5-10 / 9 / 1990$ & $\mathrm{Z} 11$ & $1.50-1.76 \mathrm{~m}$ & Charcoal & -25.00 & $714 \pm 32$ & $\begin{array}{l}675-653 \\
708-563\end{array}$ & $\begin{array}{l}1275-1297 \mathrm{AD} \\
1242-1387 \mathrm{AD}\end{array}$ & $\begin{array}{l}68.3 \% \\
95.4 \%\end{array}$ \\
\hline DEM-359 & $2 / 7 / 1993$ & H6, Pass 3, Layer B & $0.38-0.53 \mathrm{~m}$ & Charcoal & -25.00 & $804 \pm 28$ & $\begin{array}{l}729-691 \\
759-671\end{array}$ & $\begin{array}{l}1221-1259 \text { AD } \\
1190-1280 A D\end{array}$ & $\begin{array}{l}68.3 \% \\
95.4 \%\end{array}$ \\
\hline DEM-141 & $28 / 8 / 1990$ & $\begin{array}{l}\text { Z11, Northwestern re- } \\
\text { gion, Heap of cemented } \\
\text { sediment }\end{array}$ & & Charcoal & -25.00 & $5485 \pm 102$ & $\begin{array}{l}6400-6180 \\
6470-5990\end{array}$ & $\begin{array}{l}4450-4230 \mathrm{BC} \\
4520-4050 \mathrm{BC}\end{array}$ & $\begin{array}{l}68.3 \% \\
95.4 \%\end{array}$ \\
\hline DEM-920 & $6 / 7 / 1992$ & $\begin{array}{l}\Lambda 8 \text {, Pass 5, Southeastern } \\
\text { region, Loose layer, Ash } \\
\text { layer II }\end{array}$ & $0.36-0.48 \mathrm{~m}$ & $\begin{array}{l}\text { Charcoal from } \\
\text { hearth }\end{array}$ & -25.00 & $6032 \pm 21$ & $\begin{array}{l}6891-6803 \\
6937-6755\end{array}$ & $\begin{array}{l}\text { 4942-4854 BC } \\
4988-4806 \mathrm{BC}\end{array}$ & $\begin{array}{l}68.3 \% \\
95.4 \%\end{array}$ \\
\hline DEM-122 & $27 / 8 / 1990$ & I10, Northwestern region & $1.25 \mathrm{~m}$ & Charcoal & -25.00 & $6221 \pm 38$ & $\begin{array}{l}7207-7027 \\
7244-7010\end{array}$ & $\begin{array}{l}5258-5078 \mathrm{BC} \\
5295-5061 \mathrm{BC}\end{array}$ & $\begin{array}{l}68.3 \% \\
95.4 \%\end{array}$ \\
\hline DEM-913 & $6 / 7 / 1992$ & $\begin{array}{l}\Lambda 8, \text { Eastern region, Loose } \\
\text { layer }\end{array}$ & $0.32 \mathrm{~m}$ & Charcoal & -25.00 & $6248 \pm 25$ & $\begin{array}{l}7238-7098 \\
7250-7030\end{array}$ & $\begin{array}{l}5289-5149 \mathrm{BC} \\
5301-5081 \mathrm{BC}\end{array}$ & $\begin{array}{l}68.3 \% \\
95.4 \%\end{array}$ \\
\hline DEM-591 & $26 / 7 / 1994$ & $\Theta 11$, Eastern region & $0.30-0.85 \mathrm{~m}$ & Charcoal & -25.00 & $6289 \pm 28$ & $\begin{array}{l}7256-7184 \\
7269-7096\end{array}$ & $\begin{array}{l}5307-5235 \mathrm{BC} \\
5320-5147 \mathrm{BC}\end{array}$ & $\begin{array}{l}68.3 \% \\
95.4 \%\end{array}$ \\
\hline
\end{tabular}


Table 1 Summary of radiocarbon dating results of samples from the Cave of Theopetra (Continued)

\begin{tabular}{|c|c|c|c|c|c|c|c|c|c|}
\hline Lab code & $\begin{array}{l}\text { Date of } \\
\text { sampling } \\
(\mathrm{d} / \mathrm{m} / \mathrm{yr})\end{array}$ & Pit & Depth (m) & Type & $\begin{array}{l}\delta^{13} \mathrm{C} \\
(\% o) \\
\end{array}$ & Age (BP) & $\begin{array}{c}\text { Calibrated age } \\
\text { (cal BP) }\end{array}$ & Calendar age & Probability \\
\hline DEM-361 & $5 / 7 / 1993$ & $\mathrm{H} 10$ & 2.19 & Charcoal & -25.00 & $6326 \pm 94$ & $\begin{array}{l}7410-7100 \\
7420-7010\end{array}$ & $\begin{array}{l}5470-5150 \mathrm{BC} \\
5480-5060 \mathrm{BC}\end{array}$ & $\begin{array}{l}68.3 \% \\
95.4 \%\end{array}$ \\
\hline DEM-916 & 2/7/1992 & $\begin{array}{l}\Lambda 8 \text {, Pass } 2 \text {, Brown } \\
\text { layer }\end{array}$ & 0.09 & Charcoal & -25.00 & $6485 \pm 51$ & $\begin{array}{l}7430-7320 \\
7480-7270\end{array}$ & $\begin{array}{l}5480-5380 \mathrm{BC} \\
5530-5330 \mathrm{BC}\end{array}$ & $\begin{array}{l}68.3 \% \\
95.4 \%\end{array}$ \\
\hline DEM-454 & 28/7/1994 & $\begin{array}{l}\text { I11, Pass } 3 \text {, Western re- } \\
\text { gion, under a ceramic } \\
\text { pot }\end{array}$ & 0.99 & Charcoal & -25.00 & $6563 \pm 68$ & $\begin{array}{l}7560-7430 \\
7570-7320\end{array}$ & $\begin{array}{l}5610-5480 \text { BC } \\
5630-5370 \text { BC }\end{array}$ & $\begin{array}{l}68.3 \% \\
95.4 \%\end{array}$ \\
\hline DEM-585 & 29/7/1994 & $\begin{array}{l}\text { I11, Southwestern re- } \\
\text { gion }\end{array}$ & 0.99 & Charcoal & -25.00 & $6660 \pm 29$ & $\begin{array}{l}7571-7506 \\
7586-7466\end{array}$ & $\begin{array}{l}5622-5557 \mathrm{BC} \\
5637-5517 \mathrm{BC}\end{array}$ & $\begin{array}{l}68.3 \% \\
95.4 \%\end{array}$ \\
\hline DEM-914 & 7/7/1992 & $\begin{array}{l}\text { I8, Pass 6, Eastern re- } \\
\text { gion, Loose layer }\end{array}$ & $0.49-0.57$ & Charcoal & -25.00 & $6842 \pm 29$ & $\begin{array}{l}7688-7617 \\
7740-7609\end{array}$ & $\begin{array}{l}5739-5668 \mathrm{BC} \\
5791-5660 \mathrm{BC}\end{array}$ & $\begin{array}{l}68.3 \% \\
95.4 \%\end{array}$ \\
\hline DEM-455 & $1 / 8 / 1994$ & I11 & $1.02-1.55$ & Charcoal & -25.00 & $6890 \pm 43$ & $\begin{array}{l}7747-7674 \\
7793-7617\end{array}$ & $\begin{array}{l}5798-5725 \text { BC } \\
5844-5668 \text { BC }\end{array}$ & $\begin{array}{l}68.3 \% \\
95.4 \%\end{array}$ \\
\hline DEM-584 & 29/7/1994 & $\begin{array}{l}\text { I11, Southwestern re- } \\
\text { gion, Neolithic depos- } \\
\text { its }\end{array}$ & 0.87 & Charcoal & -25.00 & $6911 \pm 32$ & $\begin{array}{l}7746-7685 \\
7787-7673\end{array}$ & $\begin{array}{l}5797-5736 \text { BC } \\
5838-5724 \text { BC }\end{array}$ & $\begin{array}{l}68.3 \% \\
95.4 \%\end{array}$ \\
\hline DEM-915 & 8/7/1992 & $\begin{array}{l}\Lambda 8 \text {, Pass } 7 \text {, Eastern re- } \\
\text { gion, Loose layer }\end{array}$ & 0.67 & Charcoal & -25.00 & $7000 \pm 22$ & $\begin{array}{l}7910-7766 \\
7927-7750\end{array}$ & $\begin{array}{l}5961-5817 \mathrm{BC} \\
5978-5801 \mathrm{BC}\end{array}$ & $\begin{array}{l}68.3 \% \\
95.4 \%\end{array}$ \\
\hline DEM-575 & 29/7/1994 & $\begin{array}{l}\text { I11, Southwestern re- } \\
\text { gion }\end{array}$ & $0.97-1.07$ & Charcoal & -25.00 & $7036 \pm 44$ & $\begin{array}{l}7929-7797 \\
7945-7751\end{array}$ & $\begin{array}{l}5980-5848 \text { BC } \\
5996-5802 \mathrm{BC}\end{array}$ & $\begin{array}{l}68.3 \% \\
95.4 \%\end{array}$ \\
\hline DEM-917 & 1/7/1993 & $\begin{array}{l}\Lambda 8 \text {, Eastern region, } \\
\text { Loose layer }\end{array}$ & 1.32 & $\begin{array}{l}\text { Charcoal } \\
\text { from hearth }\end{array}$ & -25.00 & $7413 \pm 33$ & $\begin{array}{l}8320-8175 \\
8335-8064\end{array}$ & $\begin{array}{l}6371-6226 \text { BC } \\
6386-6115 \text { BC }\end{array}$ & $\begin{array}{l}68.3 \% \\
95.4 \%\end{array}$ \\
\hline DEM-919 & $2 / 7 / 1992$ & $\Lambda 8$, Pass 2, Loose layer & $0.12-0.15$ & $\begin{array}{l}\text { Charcoal } \\
\text { from hearth }\end{array}$ & -25.00 & $7456 \pm 42$ & $\begin{array}{l}8329-8201 \\
8350-8180\end{array}$ & $\begin{array}{l}6380-6252 \text { BC } \\
6401-6231 \text { BC }\end{array}$ & $\begin{array}{l}68.3 \% \\
95.4 \%\end{array}$ \\
\hline DEM-918 & $6 / 7 / 1992$ & $\begin{array}{l}\Lambda 8 \text {, Pass } 4 \text {, Brown } \\
\text { layer }\end{array}$ & $0.24-0.37$ & Charcoal & -25.00 & $7901 \pm 29$ & $\begin{array}{l}8767-8612 \\
8977-8598\end{array}$ & $\begin{array}{l}\text { 6818-6663 BC } \\
7028-6649 \text { ВC }\end{array}$ & $\begin{array}{l}68.3 \% \\
95.4 \%\end{array}$ \\
\hline DEM-360 & 19/7/1993 & H6, Pass 10, Layer B & $1.15-1.23$ & $\begin{array}{l}\text { Charcoal } \\
\text { from hearth }\end{array}$ & -25.00 & $7995 \pm 73$ & $\begin{array}{l}9010-8730 \\
9030-8610\end{array}$ & $\begin{array}{l}7060-6780 \text { BC } \\
7080-6660 \text { BC }\end{array}$ & $\begin{array}{l}68.3 \% \\
95.4 \%\end{array}$ \\
\hline DEM-583 & 28/7/1994 & $\begin{array}{l}\text { I1 1, Pass 3, Western re- } \\
\text { gion, Neolithic-Me- } \\
\text { solithic boundary }\end{array}$ & 0.57 & $\begin{array}{l}\text { Charcoal } \\
\text { from hearth }\end{array}$ & -25.00 & $8014 \pm 49$ & $\begin{array}{l}9009-8778 \\
9022-8655\end{array}$ & $\begin{array}{l}7060-6829 \mathrm{BC} \\
7073-6706 \mathrm{BC}\end{array}$ & $\begin{array}{l}68.3 \% \\
95.4 \%\end{array}$ \\
\hline DEM-576 & 2/8/1994 & $\begin{array}{l}\text { I11, Pass } 3 \text { Neolithic- } \\
\text { Mesolithic boundary }\end{array}$ & 0.77 & $\begin{array}{l}\text { Charcoal } \\
\text { from hearth }\end{array}$ & -25.00 & $8060 \pm 32$ & $\begin{array}{l}9027-8815 \\
9086-8778\end{array}$ & $\begin{array}{l}7078-6866 \text { BC } \\
7137-6829 \text { BC }\end{array}$ & $\begin{array}{l}68.3 \% \\
95.4 \%\end{array}$ \\
\hline CAMS-21733 & 8/7/1993 & $\begin{array}{l}\text { H6, Human skeleton, } \\
\text { burial in situ }\end{array}$ & 0.30 & Human bones & & $8070 \pm 60$ & $\begin{array}{l}9130-8780 \\
9250-8720\end{array}$ & $\begin{array}{l}7180-6830 \text { BC } \\
7300-6770 \text { BC }\end{array}$ & $\begin{array}{l}68.3 \% \\
95.4 \%\end{array}$ \\
\hline
\end{tabular}


Table 1 Summary of radiocarbon dating results of samples from the Cave of Theopetra (Continued)

\begin{tabular}{|c|c|c|c|c|c|c|c|c|c|}
\hline Lab code & $\begin{array}{l}\text { Date of } \\
\text { sampling } \\
(\mathrm{m} / \mathrm{d} / \mathrm{yr})\end{array}$ & Pit & Depth (m) & Type & $\begin{array}{l}\delta^{13} \mathrm{C} \\
(\% o)\end{array}$ & Age (BP) & $\begin{array}{l}\text { Calibrated age } \\
\quad(\text { cal BP) }\end{array}$ & Calendar age & Probability \\
\hline DEM-120 & $24 / 8 / 1990$ & I10 Eastern region & $1.04-1.17$ & $\begin{array}{l}\text { Charcoal from } \\
\text { hearth }\end{array}$ & -25.00 & $8524 \pm 57$ & $\begin{array}{l}9540-9490 \\
9600-9430\end{array}$ & $\begin{array}{l}7590-7540 \mathrm{BC} \\
7650-7480 \mathrm{BC}\end{array}$ & $\begin{array}{l}68.3 \% \\
95.4 \%\end{array}$ \\
\hline DEM-578 & $18 / 10 / 1994$ & $\begin{array}{l}\text { I11, Pass } 8 \text {, } \\
\text { South-eastern re- } \\
\text { gion }\end{array}$ & 1.37 & $\begin{array}{l}\text { Charcoal from } \\
\text { hearth }\end{array}$ & -25.00 & $8547 \pm 71$ & $\begin{array}{l}9600-9470 \\
9700-9430\end{array}$ & $\begin{array}{l}7650-7520 \mathrm{BC} \\
7750-7480 \mathrm{BC}\end{array}$ & $\begin{array}{l}68.3 \% \\
95.4 \%\end{array}$ \\
\hline DEM-587 & $1 / 8 / 1994$ & $\begin{array}{l}\text { I1 1, Layer 1, Cen- } \\
\text { tral region }\end{array}$ & $0.60-0.71$ & $\begin{array}{l}\text { Charcoal from } \\
\text { hearth }\end{array}$ & -25.00 & $8558 \pm 37$ & $\begin{array}{l}9548-9498 \\
9590-9478\end{array}$ & $\begin{array}{l}7599-7549 \text { BC } \\
7641-7529 \text { BC }\end{array}$ & $\begin{array}{l}68.3 \% \\
95.4 \%\end{array}$ \\
\hline DEM-125 & 28/8/1990 & $\begin{array}{l}\text { I10, Western re- } \\
\text { gion }\end{array}$ & 1.56 & $\begin{array}{l}\text { Charcoal from } \\
\text { hearth }\end{array}$ & -25.00 & $8673 \pm 76$ & $\begin{array}{l}9730-9540 \\
9900-9530\end{array}$ & $\begin{array}{l}7780-7590 \mathrm{BC} \\
7950-7580 \mathrm{BC}\end{array}$ & $\begin{array}{l}68.3 \% \\
95.4 \%\end{array}$ \\
\hline DEM-589 & $17 / 10 / 1994$ & I11, Pass 6 & 1.18 & $\begin{array}{l}\text { Charcoal from } \\
\text { hearth }\end{array}$ & -25.00 & $8863 \pm 119$ & $\begin{array}{l}10,160-9780 \\
10,210-9600\end{array}$ & $\begin{array}{l}8210-7830 \mathrm{BC} \\
8270-7650 \mathrm{BC}\end{array}$ & $\begin{array}{l}68.3 \% \\
95.4 \%\end{array}$ \\
\hline DEM-207 & $30-29 / 7 / 1991$ & $\Gamma 9$ & 2.13 & $\begin{array}{l}\text { Charcoal from } \\
\text { hearth }\end{array}$ & -25.00 & $9093 \pm 550$ & $\begin{array}{l}11,070-9540 \\
12,080-8770\end{array}$ & $\begin{array}{r}9120-7590 \mathrm{BC} \\
10,130-6820 \mathrm{BC}\end{array}$ & $\begin{array}{l}68.3 \% \\
95.4 \%\end{array}$ \\
\hline DEM-590 & $17-18 / 10 / 1994$ & I11, Pass 7 & 1.27 & $\begin{array}{l}\text { Charcoal from } \\
\text { hearth }\end{array}$ & -25.00 & $9150 \pm 112$ & $\begin{array}{l}10,480-10,210 \\
10,670-10,030\end{array}$ & $\begin{array}{l}8530-8270 \text { BC } \\
8720-7970 \text { BC }\end{array}$ & $\begin{array}{l}68.3 \% \\
95.4 \%\end{array}$ \\
\hline DEM-586 & $1 / 8 / 1994$ & I1 1, Layer 2 & 0.80 & $\begin{array}{l}\text { Charcoal from } \\
\text { hearth }\end{array}$ & -25.00 & $9188 \pm 86$ & $\begin{array}{l}10,470-10,240 \\
10,560-10,200\end{array}$ & $\begin{array}{l}8520-8290 \text { BC } \\
8620-8250 \text { BC }\end{array}$ & $\begin{array}{l}68.3 \% \\
95.4 \%\end{array}$ \\
\hline DEM-315 & 8/7/1993 & $\begin{array}{l}\text { H6, Layer B, On } \\
\text { human skeleton }\end{array}$ & 0.73 & Charcoal & -25.00 & $9274 \pm 75$ & $\begin{array}{l}10,560-10,290 \\
10,670-10,240\end{array}$ & $\begin{array}{l}8610-8340 \text { BC } \\
8720-8290 \text { BC }\end{array}$ & $\begin{array}{l}68.3 \% \\
95.4 \%\end{array}$ \\
\hline DEM-316 & $8 / 7 / 1993$ & $\begin{array}{l}\text { H6, Layer B, On } \\
\text { human skeleton }\end{array}$ & 0.73 & Charcoal & -25.00 & $9348 \pm 84$ & $\begin{array}{l}10,690-10,420 \\
11,040-10,250\end{array}$ & $\begin{array}{l}8740-8470 \text { BC } \\
9090-8300 \text { BC }\end{array}$ & $\begin{array}{l}68.3 \% \\
95.4 \%\end{array}$ \\
\hline DEM-577 & $4 / 8 / 1994$ & I11 & 1.37 & $\begin{array}{l}\text { Charcoal from } \\
\text { hearth }\end{array}$ & -25.00 & $9370 \pm 93$ & $\begin{array}{l}10,730-10,420 \\
11,060-10,250\end{array}$ & $\begin{array}{l}8780-8470 \text { BC } \\
9110-8300 \text { BC }\end{array}$ & $\begin{array}{l}68.3 \% \\
95.4 \%\end{array}$ \\
\hline DEM-588 & $3 / 8 / 1994$ & $\mathrm{I} 11$ & 1.23 & $\begin{array}{l}\text { Charcoal from } \\
\text { hearth }\end{array}$ & -25.00 & $9461 \pm 129$ & $\begin{array}{l}11,070-10,550 \\
11,160-10,300\end{array}$ & $\begin{array}{l}9120-8610 \text { BC } \\
9220-8350 \text { BC }\end{array}$ & $\begin{array}{l}68.3 \% \\
95.4 \%\end{array}$ \\
\hline DEM-142 & $24 / 8 / 1990$ & $\begin{array}{l}\text { I10, Eastern re- } \\
\text { gion }\end{array}$ & 1.17 & $\begin{array}{l}\text { Charcoal from } \\
\text { hearth }\end{array}$ & -25.00 & $9721 \pm 390$ & $\begin{array}{l}11,890-10,500 \\
12,630-10,150\end{array}$ & $\begin{array}{r}9940-8550 \mathrm{BC} \\
10,690-8210 \mathrm{BC}\end{array}$ & $\begin{array}{l}68.3 \% \\
95.4 \%\end{array}$ \\
\hline DEM-249 & $13-16 / 7 / 1992$ & $\mathrm{H} 7-\mathrm{H} 8$ & $2.02-2.22$ & Charcoal from infill & -25.00 & $10971 \pm 87$ & $\begin{array}{l}13,130-12,910 \\
13,170-12,660\end{array}$ & $\begin{array}{l}11,180-10,970 \text { ВC } \\
11,220-10,710 \text { ВC }\end{array}$ & $\begin{array}{l}68.3 \% \\
95.4 \%\end{array}$ \\
\hline DEM-248 & $8-9 / 7 / 1992$ & $\mathrm{H} 7-\mathrm{H} 8$ & $1.55-1.67$ & $\begin{array}{l}\text { Charcoal } \\
\text { from hearth }\end{array}$ & -25.00 & $11882 \pm 86$ & $\begin{array}{l}14,080-13,650 \\
14,270-13,500\end{array}$ & $\begin{array}{l}12,130-11,700 \mathrm{BC} \\
12,320-11,550 \mathrm{BC}\end{array}$ & $\begin{array}{l}68.3 \% \\
95.4 \%\end{array}$ \\
\hline DEM-245 & $14-19 / 8 / 1991$ & E12-Z12 & $1.81-1.98$ & $\begin{array}{l}\text { Charcoal } \\
\text { from hearth }\end{array}$ & 25.00 & $12045 \pm 64$ & $\begin{array}{l}14,290-13,830 \\
15,230-13670\end{array}$ & $\begin{array}{l}12,340-11,880 \mathrm{BC} \\
13,280-11,720 \mathrm{BC}\end{array}$ & $\begin{array}{l}68.3 \% \\
95.4 \%\end{array}$ \\
\hline DEM-246 & 22/8/1991 & E12-Z12 & $2.06-2.13$ & $\begin{array}{l}\text { Charcoal } \\
\text { from hearth }\end{array}$ & -25.00 & $12055 \pm 95$ & $\begin{array}{l}14,920-13820 \\
15,290-13660\end{array}$ & $\begin{array}{l}12,970-11,870 \mathrm{BC} \\
13,340-11,710 \mathrm{BC}\end{array}$ & $\begin{array}{l}68.3 \% \\
95.4 \%\end{array}$ \\
\hline
\end{tabular}


Table 1 Summary of radiocarbon dating results of samples from the Cave of Theopetra (Continued)

\begin{tabular}{|c|c|c|c|c|c|c|c|c|c|}
\hline Lab code & $\begin{array}{l}\text { Date of } \\
\text { sampling } \\
(\mathrm{m} / \mathrm{d} / \mathrm{yr})\end{array}$ & Pit & Depth (m) & Type & $\begin{array}{l}\delta^{13} \mathrm{C} \\
(\% o)\end{array}$ & Age (BP) & $\begin{array}{l}\text { Calibrated age } \\
\quad(\text { cal BP) }\end{array}$ & Calendar age & Probability \\
\hline DEM-208 & $31 / 7 / 1991$ & $\Gamma 9$ & 2.29 & $\begin{array}{l}\text { Charcoal } \\
\text { from hearth }\end{array}$ & -25.00 & $12,539 \pm 200$ & $\begin{array}{l}15,430-14,270 \\
15,630-14,110\end{array}$ & $\begin{array}{l}13,480-12,320 \mathrm{BC} \\
13,680-12,170 \mathrm{BC}\end{array}$ & $\begin{array}{l}68.3 \% \\
95.4 \%\end{array}$ \\
\hline DEM-241 & $21 / 8 / 1990$ & $\begin{array}{l}\text { K10, Northwestern } \\
\text { region, Human skele- } \\
\text { ton }\end{array}$ & 1.74 & Human bones & -21.00 & $13,723 \pm 60$ & $\begin{array}{l}16,710-16,240 \\
16,940-16,000\end{array}$ & $\begin{array}{l}14,760-14,290 \mathrm{BC} \\
14,990-14,060 \mathrm{BC}\end{array}$ & $\begin{array}{l}68.3 \% \\
95.4 \%\end{array}$ \\
\hline DEM-372 & 6/7/1993 & Z7, Layers 13-14 & $3.62-3.77$ & Charcoal from infill & -25.00 & $14,895 \pm 181$ & $\begin{array}{l}18,150-17,490 \\
18,490-17,190\end{array}$ & $\begin{array}{l}16,200-15,540 \mathrm{BC} \\
16,540-15,240 \text { ВC }\end{array}$ & $\begin{array}{l}68.3 \% \\
95.4 \%\end{array}$ \\
\hline DEM-254 & $16 / 7 / 1992$ & $\Theta 10$ & $3.36-3.61$ & Charcoal from infill & -25.00 & $16,540 \pm 98$ & $\begin{array}{l}20,040-19,390 \\
20,350-19,090\end{array}$ & $\begin{array}{l}18,090-17,440 \mathrm{BC} \\
18,400-17,140 \mathrm{BC}\end{array}$ & $\begin{array}{l}68.3 \% \\
95.4 \%\end{array}$ \\
\hline DEM-61 & $\begin{array}{l}26-27 / 10 / \\
1988\end{array}$ & $\mathrm{Z} 8$ & $3.62-3.83$ & $\begin{array}{l}\text { Charcoal } \\
\text { from hearth }\end{array}$ & -25.00 & $25,354 \pm 2132$ & $29,755 \pm 2132^{\mathrm{a}}$ & $29,940-25,670 \mathrm{BC}^{\mathrm{a}}$ & $68.3 \%$ \\
\hline DEM-223 & 23/8/1991 & $\Theta 10-\mathrm{I} 10$ & $2.92-3.29$ & $\begin{array}{l}\text { Charcoal } \\
\text { from hearth }\end{array}$ & -25.00 & $30,023 \pm 876$ & $34,997 \pm 876^{a}$ & $33,920-32,170 \mathrm{BC}^{\mathrm{a}}$ & $68.3 \%$ \\
\hline DEM-374 & $5-7 / 7 / 1993$ & Z7, Layer 13 & $3.62-3.77$ & $\begin{array}{l}\text { Charcoal } \\
\text { from hearth }\end{array}$ & -25.00 & $32,672 \pm 1503$ & $37,913 \pm 1503^{*}$ & $37,470-34,460 \mathrm{BC}^{\mathrm{a}}$ & $68.3 \%$ \\
\hline DEM-247 & $14 / 8 / 1991$ & $\Theta 10$ & $3.03 \mathrm{~m}$ & $\begin{array}{l}\text { Charcoal } \\
\text { from hearth }\end{array}$ & -25.00 & $33,085 \pm 1573$ & $38,364 \pm 1573^{a}$ & $37,990-34,840 \mathrm{BC}^{\mathrm{a}}$ & $68.3 \%$ \\
\hline DEM-134 & $8 / 1990$ & Z8-Z9 & $4.55 \mathrm{~m}$ & $\begin{array}{l}\text { Charcoal } \\
\text { from hearth }\end{array}$ & -25.00 & $36,827 \pm 845$ & $39,177 \pm 845^{\mathrm{b}}$ & $38,070-36,380 \mathrm{BC}^{\mathrm{b}}$ & $68.3 \%$ \\
\hline DEM-74 & $18 / 7 / 1989$ & Z9 & $4.28 \mathrm{~m}$ & $\begin{array}{l}\text { Charcoal } \\
\text { from hearth }\end{array}$ & -25.00 & $38,079 \pm 1942$ & $40,380 \pm 1942^{b}$ & $40,370-36,490 \mathrm{BC}^{\mathrm{b}}$ & $68.3 \%$ \\
\hline DEM-133 & $24 / 8 / 1990$ & Z8-Z9 & $4.39-4.49$ & $\begin{array}{l}\text { Charcoal } \\
\text { from hearth }\end{array}$ & -25.00 & $39,274 \pm 4771$ & $41,370 \pm 4771^{\mathrm{b}}$ & $44,190-34,650 \mathrm{BC}^{\mathrm{b}}$ & $68.3 \%$ \\
\hline DEM-140 & $8 / 1990$ & $\mathrm{Z} 8$ & $4.80 \mathrm{~m}$ & $\begin{array}{l}\text { Charcoal } \\
\text { from hearth }\end{array}$ & -25.00 & $39,414 \pm 3914$ & $41,410 \pm 3914^{b}$ & $43,370-35,550 \mathrm{BC}^{\mathrm{b}}$ & $68.3 \%$ \\
\hline DEM-613 & $11 / 11 / 1996$ & $\begin{array}{l}\Theta 10 \text {, Human foot- } \\
\text { prints layer }\end{array}$ & $4.07-4.17$ & $\begin{array}{l}\text { Charcoal } \\
\text { from hearth }\end{array}$ & -25.00 & $46,327 \pm 1590$ & $46,330 \pm 1590^{\mathrm{b}}$ & $45,970-42,790 \mathrm{BC}^{\mathrm{b}}$ & $68.3 \%$ \\
\hline
\end{tabular}

${ }^{a}$ Calibrated approximately according to Bard et al. (1998). See text.

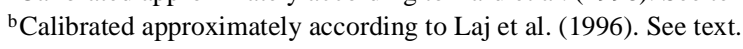




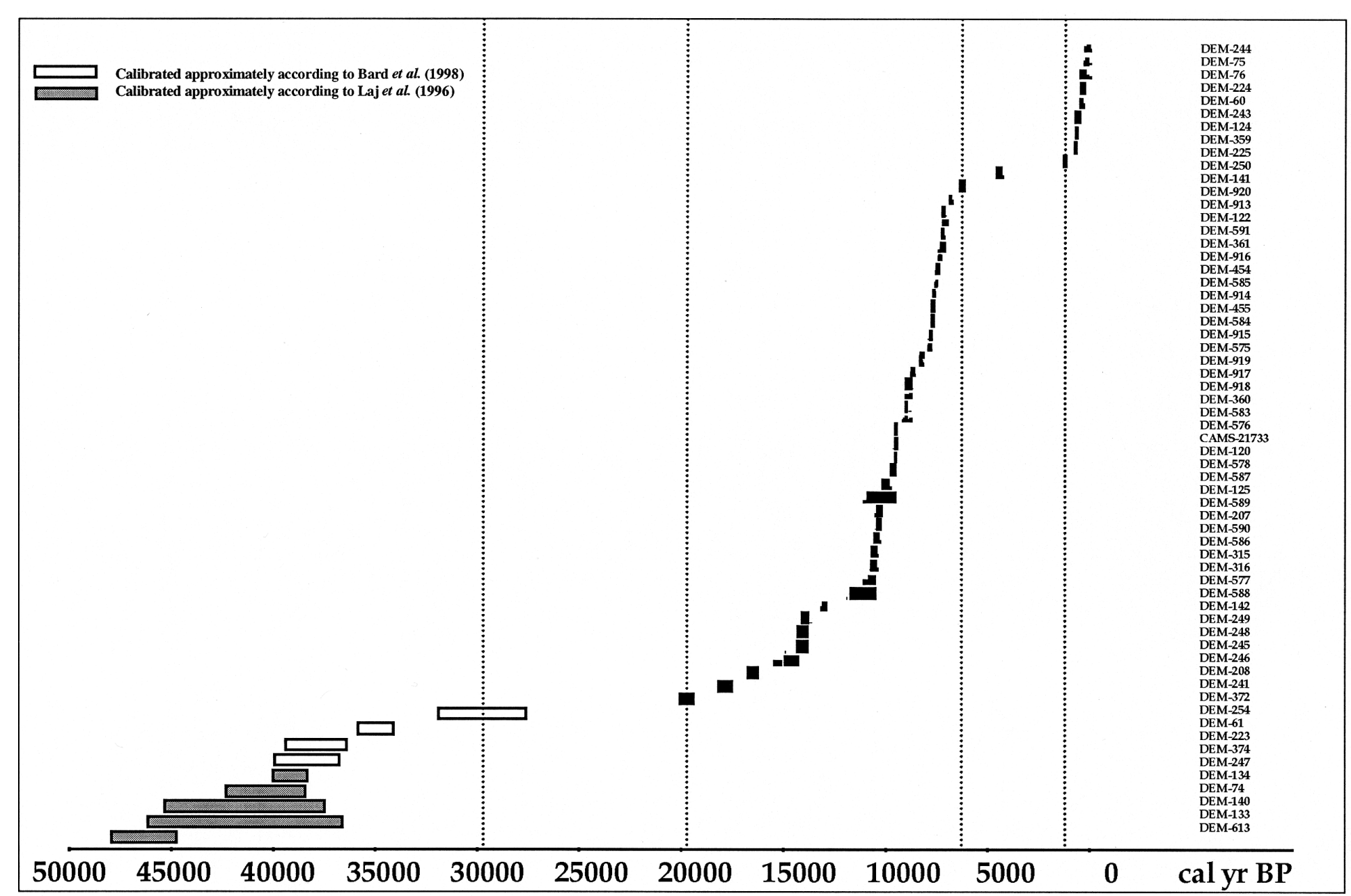

Figure 3 The distribution of the calibrated dates of the samples ranging from $46,000 \mathrm{BP}(\approx 48,000 \mathrm{cal}$ yr BP) up to the present sorted by age. The length of the bars represents the age range; the height represents the percent probability that the sample lies in the specific range. 
dropping from the cave's roof. Apparently, trapping by calcite crystallization was the only way for the Late Neolithic deposits to survive the successive flooding by the ground waters. In the future, the dating of charcoal samples retrieved from the core of such heaps could produce more ages from the Late Neolithic period, which are relatively rare.

An attempt was made to provide information on the sedimentation rate using the time interval versus the depth at consecutive layers bearing traces of human activity (hearths). For this purpose two trenches perpendicular to each other (pits Z7-Z8-Z9-Z10-Z11-Z12 and @10-I10-K10) near the central area of the cave (Figure 2) were selected. Figures 4 and 5 show the correlation of the calibrated radiocarbon ages of charcoal samples, originating from hearths found in these two trenches. The ages span from 46,000 to present and 46,000 to 5000 cal BP, respectively, versus the corresponding depth from surface. The slope of the line of the best linear fit in both cases is found to be about the same. This means that the sedimentation rate was rather slow reaching $0.8 \mathrm{~cm} / 100 \mathrm{yr}$ in the area of the trench Z7-Z8-Z9-Z10-Z11-Z12 and $0.7 \mathrm{~cm} / 100 \mathrm{yr}$ in the area of the trench $\Theta 10-\mathrm{I} 10-\mathrm{K} 10$. Contrary to that, near the eastern walls of the cave (pit $\Lambda 8$ ) the sedimentation rate was found much faster during the Early and the Middle Neolithic period reaching $10.7 \mathrm{~cm} / 100 \mathrm{yr}$ (Figure 6). This may be explained by the climatic change in the Holocene. These results are tentative because there are many factors, which cannot be controlled, such as sediment shrinkage, erosion, human intervention, etc. However, the curves show a great difference in the sedimentation rate between the different periods of time.

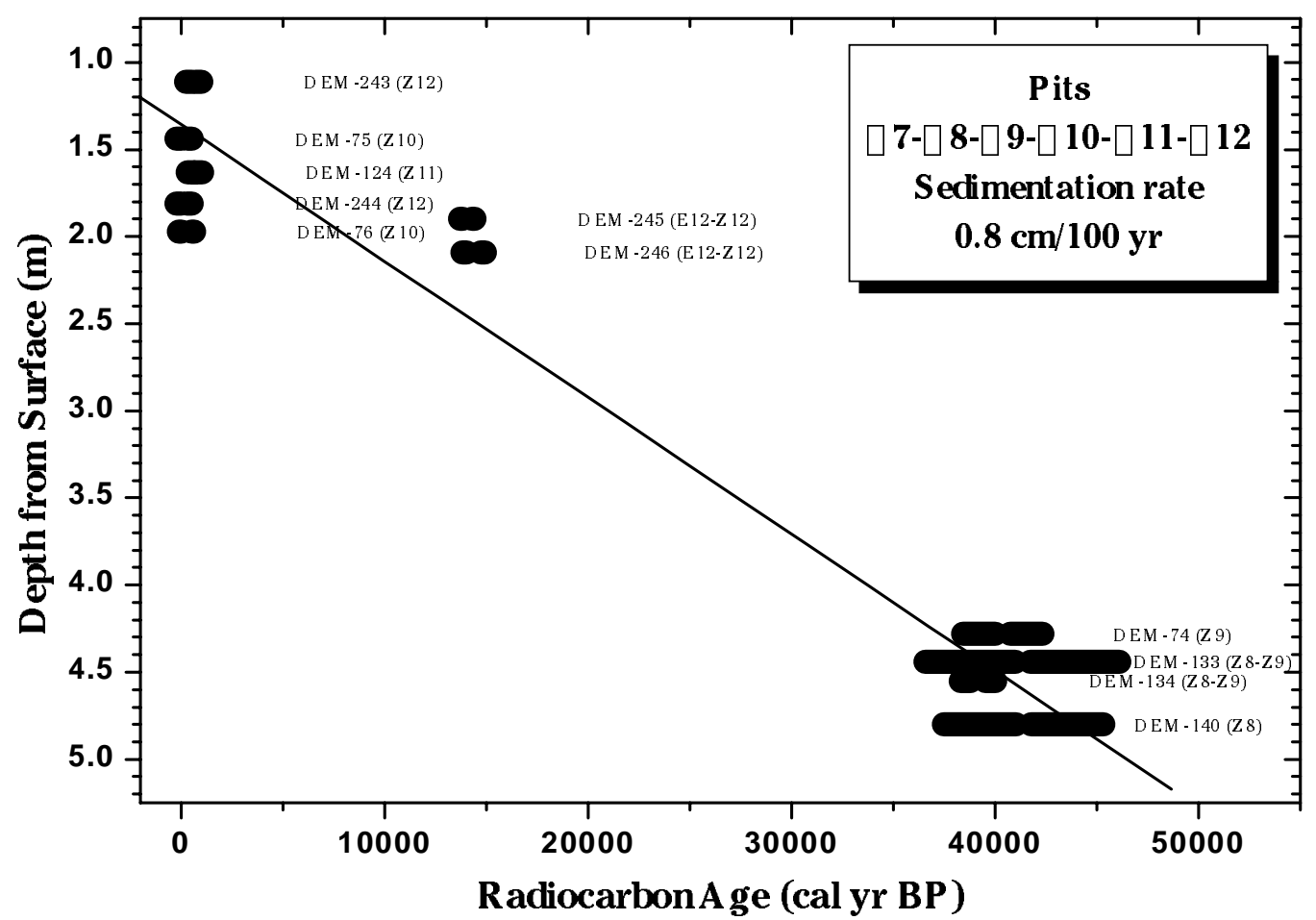

Figure 4 Calibrated dates versus depth of samples originating from the region of the pits $\Theta 10-\mathrm{I} 10$-K10 to determine the sedimentation rate of the sediments, which were carried in the cave by the ground waters. 
Special attention was given to date accurately the transition from the Mesolithic to the Neolithic period in the Theopetra cave. For that purpose 18 charcoal samples were dated from the two neighboring pits I10 and I11 originating from a deposit $1 \mathrm{~m}$ thick in a relatively small depth from the present surface (0.57-1.56 m depth). Figure 7 shows the correlation of the calibrated radiocarbon ages versus the corresponding depth. According to the archaeological and geological data on the cave stratigraphy the end of the Mesolithic period is defined by the samples DEM-583 and DEM576, which were collected precisely at the boundary between the Mesolithic and the Neolithic deposits of the pit I11 (Kyparissi-Apostolika 2000a). The average age of these samples puts the transition at $8910 \pm 120$ cal BP, continuous vertical line in Figure 6. In the same figure the dashed lines indicate the time boundaries of next cultural phases of the Neolithic period (Late and Middle Neolithic), as they have resulted from earlier archaeological research in Thessaly (Gallis 1996). The large scattering of the ages with depth, which is observed in Figure 6, is probably due to the combined action of the three following factors: 1) the anthropogenic activity during the Neolithic period when, according to the archaeological data, pits were dug out disturbing the preceding mesolithic layers, 2) similar anthropogenic activity during the historical times and finally, and 3) the action of the ground waters inside the cave during the Neolithic and younger periods.

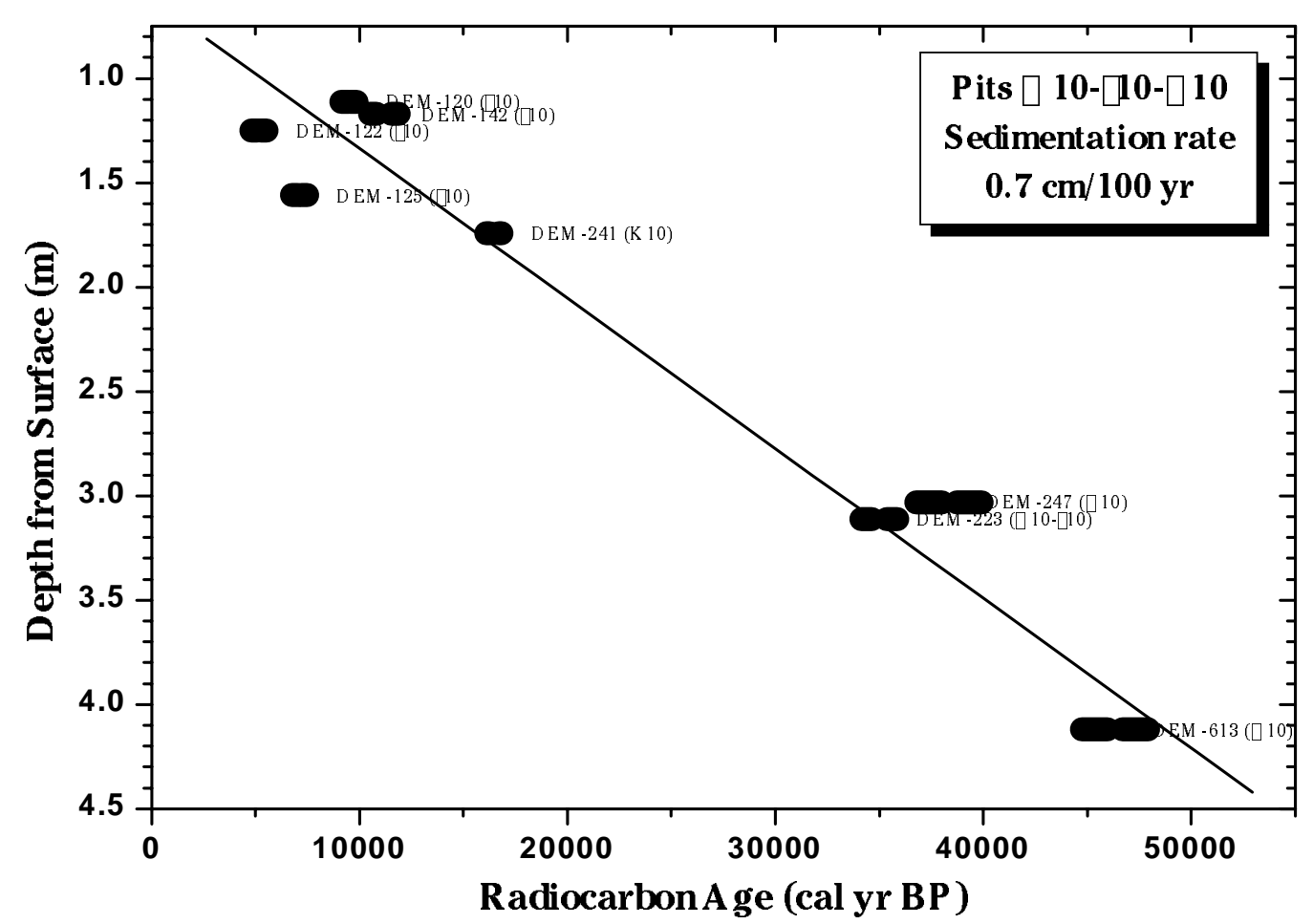

Figure 5 Calibrated dates versus depth of samples originating from the region of the pits Z7-Z8-Z9-Z10-Z11-Z12 in order to determine the sedimentation rate of the sediments, which were carried in the cave by the ground waters.

\section{Dating of the Human Skeletons}

Two human skeletons (Stravopodi et al. 1999) have been discovered so far in Theopetra cave, in the pits K10 and H6 (Figure 2). Both skeletons were very well preserved as they were found at a part of 


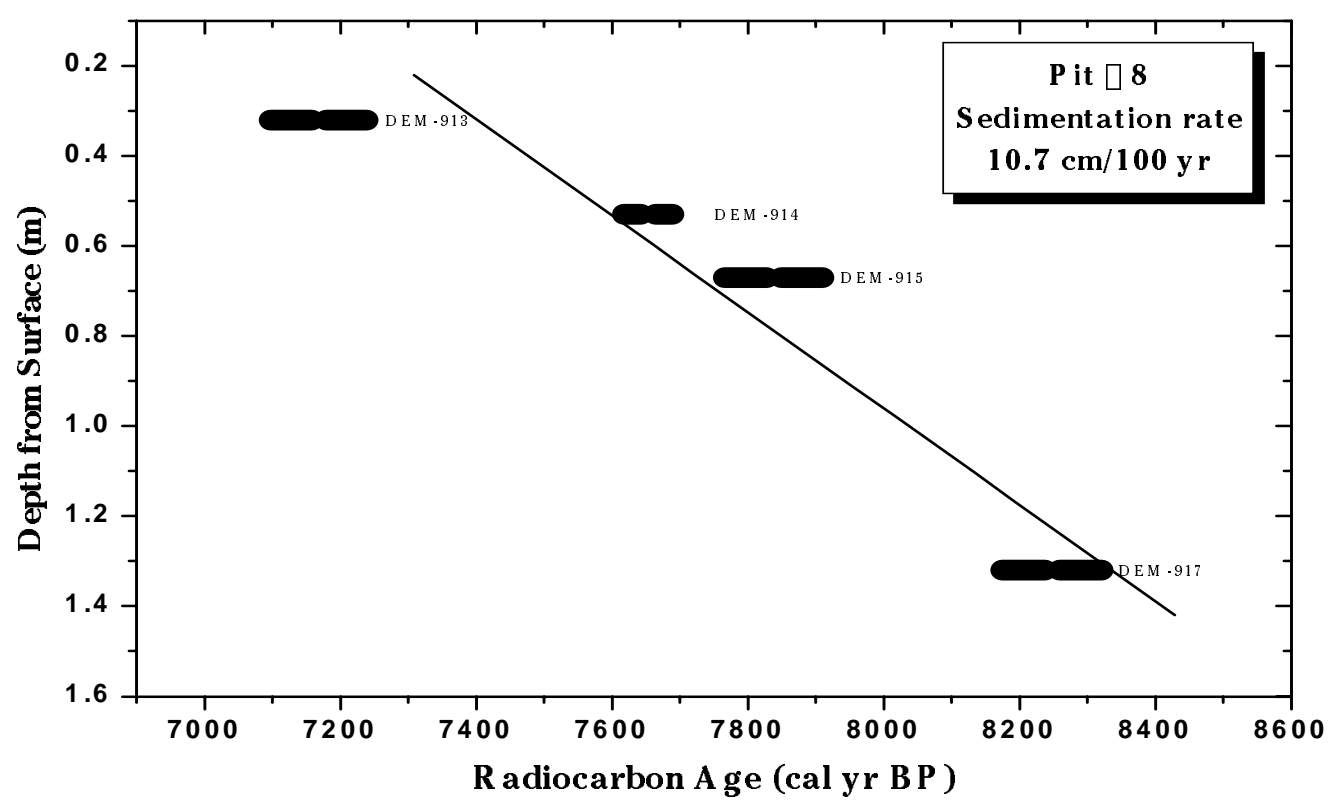

Figure 6 Calibrated dates versus depth of samples originating from the region of the pit $\Lambda 8$ in order to determine the sedimentation rate of the sediments during the Early and the Middle Neolithic period near the eastern walls of the cave.

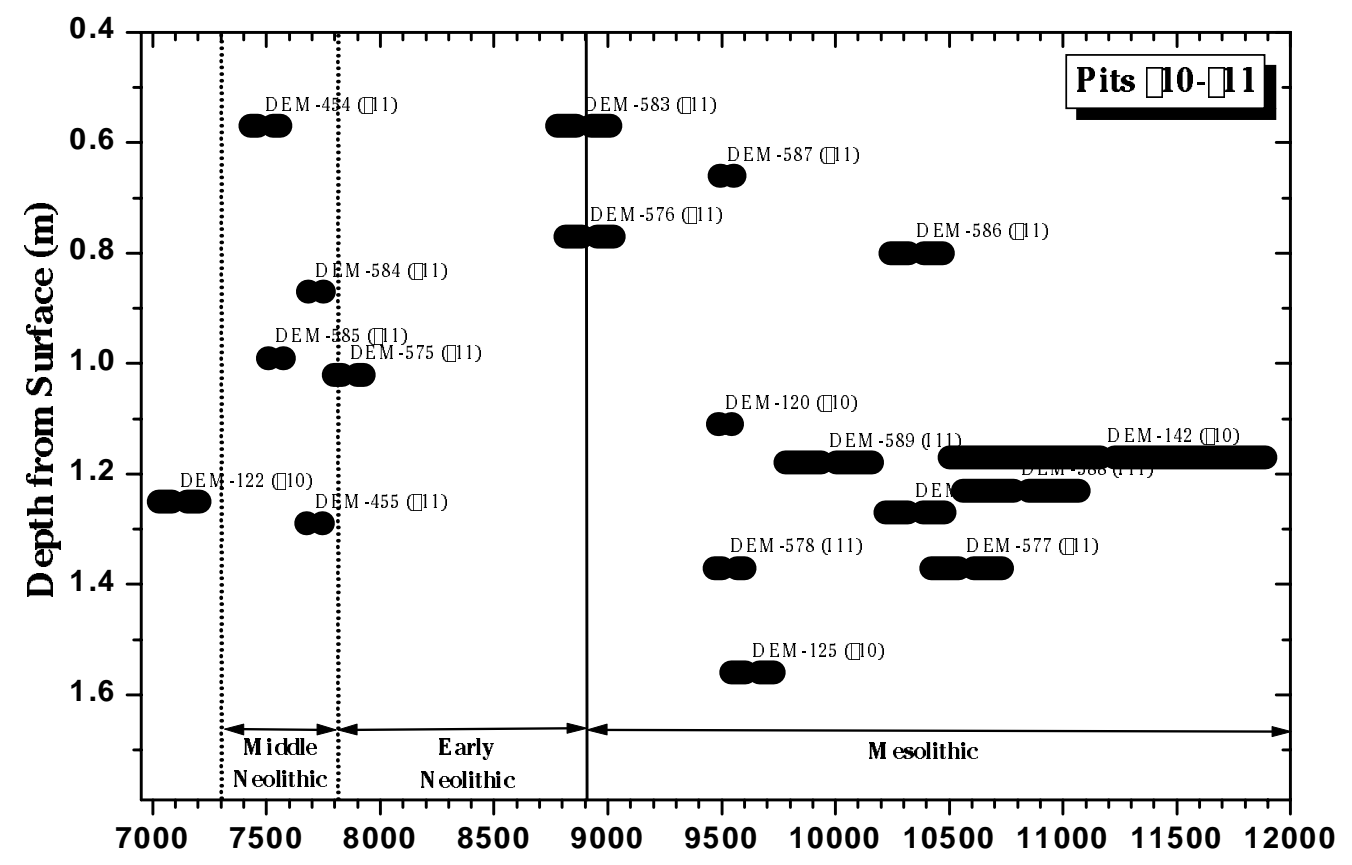

Figure 7 Calibrated dates versus depth of samples originating from the region of the pits I10-I11 in the cave. The ages of the samples DEM-583 and DEM-576, which according to the archaeological and the geological data define the boundary between Mesolithic and Neolithic period, give a mean value $8910 \pm 120$ cal BP, which defines this transition. 
the cave (eastern and central to the north), where the diagenesis process was not very severe (Karkanas and Weiner 2000).

The first skeleton was found smashed by illegal excavators in pit K10. This unfortunate incident did not allow for a safe conclusion of whether or not it was a burial as the indications suggest. The skeleton was dated using $154 \mathrm{~g}$ of collagen from the long bones. The collagen was extracted after prolonged soaking of the bone pieces into a $\mathrm{HCl}$ acid solution (Facorellis 1996). The result of the ${ }^{14} \mathrm{C}$ dating (DEM-241) showed that it belonged to a human who lived during the Upper Paleolithic period, $16475 \pm 235$ cal BP (Table 1).

The second skeleton was found in an undisturbed burial in-situ in a hole dug in the pit H6. The biometric analysis showed that it belonged to a woman (Stravopodi et al. 1999). Due to the importance and rarity of the find, initially it was considered necessary to preserve it intact in order to exhibit it in the future. So, it was decided not to take sample from the bones for dating but to make an attempt to date it indirectly. For that purpose two charcoal samples found in contact with the skeleton were collected for dating. The ages of these two samples DEM-315: $10425 \pm 135$ cal BP and DEM-316: $10555 \pm 135$ cal BP are overlapped within 1 standard deviation (Table 1).

However, for the benefit of the scientific research a very small fragment of a cranial bone measuring a surface of about $1 \mathrm{~m}^{2}$ was finally sent to E Nelson (1996) at the Department of Archaeology, Simon Fraser University at Burnaby Canada who separated and isolated the high-molecular weight collagen fraction. Then this fraction was shipped to the Center for Accelerator Mass Spectrometry at the Lawrence-Livermore National Laboratory for AMS ${ }^{14} \mathrm{C}$ measurement. The ${ }^{14} \mathrm{C}$ age of the sample (CAMS-21773) is $8070 \pm 60 \mathrm{BP}$ and the corresponding calibrated age is $8955 \pm 175 \mathrm{cal} \mathrm{BP}$ (probability $68.3 \%$, Table 1) suggesting that it belonged to a human who lived at the end of the Mesolithic period. The stable isotope ratio, as well as the $\mathrm{C} / \mathrm{N}$ ratio were measured at the Department of Oceanography, University of British Columbia and found to be $\delta^{13} \mathrm{C}=-20.5 \%$ and $\mathrm{C} / \mathrm{N}=3.1$, respectively.

By comparing the dating results of the charcoal samples in contact with the skeleton DEM-315 and DEM-316 and the age of the bones (CAMS-21773) one can see that the charcoal samples are older by about 1500 years. Apparently, during the initial digging of the hole to bury the body, the older layers underneath, obviously containing charcoal pieces, were dug out and then used to cover the body.

\section{Dating of the Human Footprints}

During the excavation campaign of 1996 four human footprints (Figure 8) preserved in a good state on a reddish clay sediment were unearthed in the pit I10 (Figure 2) at a depth slightly lower than $4 \mathrm{~m}$. Numerous remains of hearths existed along the layer whose black color presents a striking contrast with the red color of the sediment. These hearths represent the earliest presence of fire in Thessaly (Kyparissi-Apostolika 2000b).

Due to the exceptional importance of the find and the absence of charcoal pieces visible to the naked eye $48 \mathrm{~g}$ of black soil were collected for radiocarbon dating from the specific layer. The sample was taken from an area in the interior of a hearth covering $50 \mathrm{~cm}^{2}$ and $0.5 \mathrm{~cm}$ thick. The sampling was performed selectively at points of that area where the black color of the soil indicated higher content in charcoal particles, thus ensuring the reliable dating of the hearth (Mook and Waterbolk 1985).

The sample was then dried and homogenized in the laboratory. A small portion of the natural sample was retained and the rest was chemically pretreated with acid-alkali-acid solutions to remove the contaminants. In a next step, two pellets were produced using high pressure from both natural and chemically pretreated parts in order to measure the percent of carbon content. The surface of the pel- 


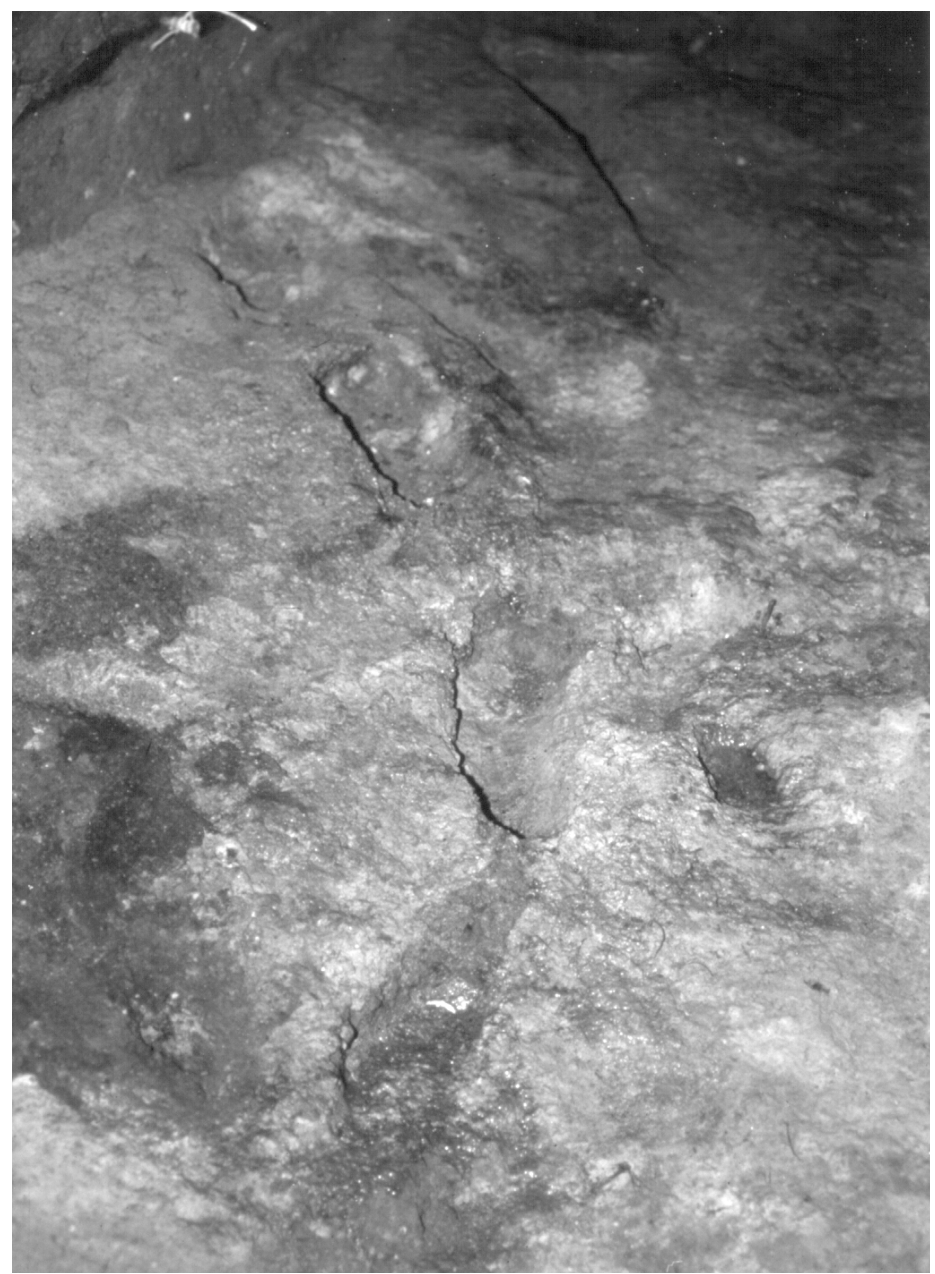

Figure 8 Human footprints uncovered at a depth of $4.10 \mathrm{~m}$ at pit $\Theta 10$ of the Theopetra cave, dated to $46,330 \pm$ $1590 \mathrm{BP}$.

lets was coated with gold to make it conductive and they were both examined under a Scanning Electron Microscope combined with Energy Disperse X-ray Analysis (SEM-EDS). The elemental quantitative analysis was performed without using the Be (Beryllium) window of the detector allowing the detection of the light elements of the Periodic Table, until the atomic number 5 (Boron). The analysis results showed that the carbon content in the natural and the chemically pretreated parts was about $35 \%$ and $50 \%$, respectively. The enrichment in carbon after the chemical pretreatment is due to the elimination of the $\mathrm{CaCO}_{3}$ and other minerals from the sample after the successive treatments with the acid and alkali solutions (Facorellis 1996).

$\mathrm{X}$-ray diffraction analysis was used on both natural and chemically pretreated parts to ensure that all $\mathrm{CaCO}_{3}$ was eliminated from the sample after the chemical pretreatment. Table 2 shows the results of the analysis. One can see that in the natural part of the sample the authigenic minerals crandallite, tinsleyite, which are formed during the diagenesis processes in Theopetra cave (Karkanas et al. 1999; Karkanas and Weiner 2000), are detected. The calcite, which is also detected in the natural sample, is probably due to the water dropping from the cave roof which permeated the clay sediment bearing the footprints after it was excavated. On the other hand, the calcite is not detected in the chemically pre- 
treated part meaning that the acid treatment was effective, though the acid seems to dissolve the crandallite mineral, which is also not detected in that part of the sample.

The carbon enriched sample, which was obtained after the chemical pre-treatment, was prepared for radiocarbon dating (DEM-613). It was measured nine times in two different gas proportional counters with a total measuring time of 22 days. The calculated conventional ${ }^{14} \mathrm{C}$ age of this sample is $46,327 \pm 1590 \mathrm{BP}$ (Table 1 ) and represents the terminus ante quem for the formation of the human footprints.

Table 2 X-ray diffraction analysis and electron probe microanalysis results of the sediment sample (DEM-613), as received, and after chemical treatment before being ${ }^{14} \mathrm{C}$ dated. The sample originates from the layer at the pit $\Theta 10$ of the cave bearing human footprints

\begin{tabular}{lll}
\hline & \multicolumn{2}{c}{ Chemical formula } \\
\cline { 2 - 3 } Mineral & Natural sample & Chemically treated sample \\
\hline Quartz & $\mathrm{SiO}_{2}$ & $\mathrm{SiO}_{2}$ \\
Crandallite & $\mathrm{CaAl}_{3}\left(\mathrm{PO}_{4}\right)_{2}(\mathrm{OH})_{5} \cdot \mathrm{H}_{2} \mathrm{O}$ & - \\
Tinsleyite & $\mathrm{KAl}_{2}\left(\mathrm{PO}_{4}\right)_{2}(\mathrm{OH}) \cdot 2 \mathrm{H}_{2} \mathrm{O}$ & $\mathrm{KAl}_{2}\left(\mathrm{PO}_{4}\right)_{2}(\mathrm{OH}) \cdot 2 \mathrm{H}_{2} \mathrm{O}$ \\
Calcite & $\mathrm{CaCO}_{3}$ & - \\
Illite & $\left(\mathrm{K}_{3} \mathrm{O}\right)_{2} \mathrm{Al}_{2} \mathrm{Si}_{3} \mathrm{AlO}_{10}(\mathrm{OH})_{2}$ & $\left(\mathrm{~K}_{3} \cdot \mathrm{H}_{3} \mathrm{O}\right) \mathrm{Al}_{2} \mathrm{Si}_{3} \mathrm{AlO}_{10}(\mathrm{OH})_{2}$ \\
Hematite & $\mathrm{Fe}_{2} \mathrm{O}_{3}$ & $\mathrm{Fe}_{2} \mathrm{O}_{3}$ \\
Albite & $\mathrm{NaAlSi}_{3} \mathrm{O}_{8}$ & $\mathrm{NaAlSi}_{3} \mathrm{O}_{8}$ \\
Microcline & $\mathrm{KAlSi}_{3} \mathrm{O}_{8}$ & $\mathrm{KAlSi} \mathrm{O}_{8}$ \\
Carbon (Electron probe microanalysis) & $\mathrm{C}(35 \%)$ & $\mathrm{C}(50 \%)$ \\
\hline
\end{tabular}

\section{ARCHAEOLOGICAL IMPLICATIONS}

The upper deposits of Theopetra cave accumulated during the Neolithic period were disturbed by natural agencies as mentioned above. Despite its current condition and being an isolated settlement at the northwestern edge of the thessalic plain, all the Neolithic phases, from the very initial Early Neolithic to the Chalcolithic, are represented with rich and impressive finds. The cave is estimated to have been abandoned at the Chalcolithic although it has never stopped being used occasionally by shepherds until recently.

The pottery is representative of all the Neolithic phases (Kyparissi-Apostolika 2000a). Although west Thessaly is mainly known for settlements belonging to the Early Neolithic (EN) period, it seems that in the case of Theopetra the richest Neolithic phases are characterized by the Middle Neolithic (MN) and the Late Neolithic (LN) pottery. The "primitive painted" pottery of Theocharis (1973) consisted of simple dark lines and red triangles straight on the unpolished surface of the vase is present here, while even more primitive monochrome and undecorated pottery probably belonging to a pre-Neolithic phase, come out from the underlying Mesolithic deposit. The typical Protosesklo and Prosesklo decoration is also present as well as the Rainbow and the Blacktopped. Incised and impressed decoration known already from other sites of the same period in Thessaly characterize the EN pottery, while a stylistic and technological evolution is obvious.

The MN pottery is represented by excellent samples of decorated vessels among which a good number of "offering tables" and decorative styles known from the rest of Thessaly and from Servia in west Macedonia (red on light with solid style, scraped ware, $\mathrm{A} 3 \beta, \mathrm{A} 3 \gamma, \mathrm{A} 3 \delta, \mathrm{A} 3 \varepsilon, \mathrm{A} 3 \zeta$ ) (Wace and Thompson 1912). 
The rich LN pottery is represented by the following categories:

1. Very thin and high temperature baked Grey on Grey, which is faced as a technological and stylistic evolution of the MN scraped ware, while the so-called Protogrey, also present here, is faced as an intermediate stage between the two above categories. They belong to Tsangli (Kyparissi-Apostolika 2000a) phase at the beginning of the LN.

2. Black Burnished ware, known as Larissa culture, in very good quality and variety of shapes. Brown and red variations are present in very good quality as well. It also belongs to Tsangli phase.

3. Matte-painted (black on white, black on red) with several decorative styles, mainly geometric, and in a good variety of shapes. It belongs to the Tsangli phase as well.

4. Multicolored (usually black and red on white) pottery of Arapi phase is present in smaller quantities.

5. Dark on white ware, characteristic of the main Dimini phases, Otzaki C (Hauptmann 1981) and $\mathrm{B} 3 \alpha 3$ (Tsountas 1908). It is represented by a rather small number of very good quality.

6. Incised decoration in well-organized decorative panels sometimes filled with white material. They characterize the Rachmani phase of the Chalcolithic period.

7. So-called rope-shaped decoration consisted of plastic strips with fingerprints on them. They usually are seen under the brims, but sometimes they cross the whole body of the jars. They characterize the Chalcolithic and the Early Bronze Age.

Apart from the pottery, worth mentioning are a few figurines-naturalistic and schematic-each a different type from the others, among which includes part of a marble figurine and an acrolith. Of special importance is a clay figurine of Balkan type and possible origin.

Equally important are the ornaments, beads, and bracelets made of the shell Spondylus gaederopus. Some of the beads are barrel-shaped and unusually large (up to $5 \mathrm{~cm}$ long and $3 \mathrm{~cm}$ thick) and it seems that they were fabricated on site by local craftsmen who had found the raw material near the aegean shores or got it as a result of intra-site exchanges. The most striking among the ornaments is a gold ring-idol, also of balkan type, well known from the cemetery of Varna in Bulgaria and from other sites of the Balkans and the east Aegean (Kyparissi-Apostolika 1999).

Spindle whorls and other implements for weaving, as well as a big quantity of milling stones for processing cereals and calorific materials, are included among the Neolithic finds of Theopetra cave.

The Mesolithic period is for the first time attested in Thessaly and represents a distinct case for the mainland Greece so far. Additionally, this period is of special importance for the transition from the Pleistocene to the Holocene and for the transition from wild to the cultivated plants, from wild to domesticated animals and for the beginning of pottery technology, setting on a new basis the problem of the "imported" Neolithic culture in Greece. The human presence during that period of time is confirmed by the recovery of the aforementioned woman's skeleton (CAMS-21733). The other human skeleton (DEM-241) reflects a man of the postglacial Upper Palaeolithic period. DNA analysis carried out on skeletal remains from the Palaeolithic, Mesolithic and Neolithic deposits showed biological relation and continuity of the population, while masses of unbaked clay found in late Upper Palaeolithic layers indicate that the knowledge of the plastic properties of this material that lead to the pottery technology was much older than was believed in the past (Evison et al. 2000).

Under the Mesolithic and the postglacial Upper Palaeolithic layers, a hard sediment was found. It has a tongue-like shape thinning from the entrance of the cave towards the center (Figure 2) (Karkanas et al. 1999). Its formation is due to diagenetic processes in that part of the cave dated after 30,000 cal BP and before 18,000 cal BP. 
A series of hearths uncovered in the horizon dated to around 30,000 cal BP reflect an increase in the population and in the human activities in the cave due to an amelioration of the climate. In general the Upper Palaeolithic layers are poor in finds compared to the other periods (Adam 1999).

The deepest 2.50-m-thick deposit represents the Middle Palaeolithic period with multiple layers of hearths alternated with water-lain sediments due to the invasion of water through the karstic acquifers during the Pleistocene. In this Middle Palaeolithic deposit many lithic artifacts of good quality show that this must have been the richest period of the Pleistocene in the cave with increased population when the climate was warm (Panagopoulou 1999). At the deepest layer where fire remains are present, dated to around 46,000 BP, human footprints were uncovered which are at present under study to identify the human type to whom they belong (Homo sapiens sapiens or neanderthalensis). An even deeper sediment without hearth traces at all is the oldest one in the cave, estimated to date up to 70,000 BP according to geological criteria. Lithic artifacts found in this sediment are technologically comparable to the Middle Palaeolithic of the overlying layers.

\section{CONCLUSIONS}

The systematic ${ }^{14} \mathrm{C}$ dating results set the bases for interpretation of the archaeological finds, as well as the geological and paleoclimatic events by researchers of all the corresponding specialities. In particular, they helped to clarify the stratigraphic anomalies and to certify the human presence in Theopetra cave from the Middle and Upper Palaeolithic, Mesolithic and Neolithic periods, until recently.

The available ${ }^{14} \mathrm{C}$ data reveal three age gaps. The first begins from around 35,000 ending at around 20,000 cal BP coinciding to the end of the last glacial maximum and it is interrupted at around $30,000 \mathrm{cal} \mathrm{BP}$ by a brief and mild climatic episode. The next one begins around 13,000 cal BP, ending around $11,200 \mathrm{cal} \mathrm{BP}$, and it is attributed to the Younger Dryas climatic event. The third age gap starts around 6300 and ends at around $1200 \mathrm{cal} \mathrm{BP}$. It is most probably due to the eroding action of the ground waters from the cave's karstic aquifers.

The use of the new international calibration curve allowed the accurate calibration of the conventional ${ }^{14} \mathrm{C}$ ages falling into the time interval from 24,000 cal BP to the present. As several samples gave ages beyond the calibration curve, an attempt was made to calibrate those samples using various approximation methods.

The systematic dating of many layers with depth allowed the calculation of the sedimentation rate in the cave. The results show two different rates. A slow one $(0.7 \mathrm{~cm} / 100 \mathrm{yr})$ in the period 5000 $46,000 \mathrm{cal} \mathrm{BP}$ and a fast one $(10.7 \mathrm{~cm} / 100 \mathrm{yr})$ in the period $8300-7100 \mathrm{cal} \mathrm{BP}$. The difference must be due to change of the climate during the Holocene.

The direct ${ }^{14} \mathrm{C}$ dating of samples from the two human skeletons places one of them to the end of the Mesolithic period ( $8955 \pm 172 \mathrm{cal} \mathrm{BP})$ and the other to the Upper Palaeolithic period (16470 \pm 235 cal BP). The transition from the Mesolithic to the Neolithic period in the cave was dated to $8910 \pm$ 120 cal BP.

The age of the soil rich in charcoal particles from a hearth found on the layer bearing the human footprints gives the terminus ante quem of their formation at $46,300 \pm 1600 \mathrm{BP}$. At the same time, it represents the earliest evidence of fire in Thessaly. 


\section{ACKNOWLEDGMENTS}

The authors would like to express their thanks to Prof E Nelson at the Department of Archaeology, Simon Fraser University at Burnaby Canada who carried out the preparation of the sample from the mesolithic skeleton for AMS dating. Many thanks are also due to the technical staff of the Laboratory of Archaeometry, NCSR "Demokritos”, K Gogidou, M Korozi, and M E Kyriazi for the sample preparation for ${ }^{14} \mathrm{C}$ dating.

\section{REFERENCES}

Adam E. 1999. Preliminary presentation of the Upper Palaeolithic and Mesolithic stone industries of Theopetra cave, Western Thessaly. British School at Athens Studies 3:266-270.

Ardaens E. 1978. Geologie de la chaine du Vardussia, comparaison avec le massif du Koziakas (Grèce Continentale). Thèse 3ème cycle. Lille, France. In French.

Arslanov KA, Svezhentsev YS. 1993. An improved method for radiocarbon dating fossil bones. Radiocarbon 35(3):387-91.

Bard E, Arnold M, Hamelin B, Tisnerat-Laborde N, Cabioch G. 1998. Radiocarbon calibration by means of mass spectrometric ${ }^{230} \mathrm{Th} /{ }^{234} \mathrm{U}$ and ${ }^{14} \mathrm{C}$ ages of corals: an updated database including samples from Barbados, Mururoa and Tahiti. Radiocarbon 40(3):108592.

Brown TA, Nelson DE, Vogel JS, Southon JR. 1988. Improved collagen extraction by modified Longin method. Radiocarbon 30(2):171-7.

Evison M, Kyparissi-Apostolika N, Stravopodi E, Fieller N, Smillie D. 2000. An ancient HLA from a Palaeolithic skeleton from Theopetra cave, Greece. In: Kyparissi-Apostolika N, editor. Proceedings of the International Scientific Conference "Theopetra caveTwelve years of Excavations and Research. Trikala, 78 Nov 1998. p 109-18.

Facorellis Y. 1996. Study of the conditions and parameters for high precision dating with ${ }^{14} \mathrm{C}$ [unpublished $\mathrm{PhD}$ thesis]. University of Patras, Greece.

Facorellis Y, Maniatis Y, Kromer B. 1997. Study of the parameters affecting the correlation of background versus cosmic radiation in $\mathrm{CO}_{2}$ counters: reliability of dating results. Radiocarbon 39(3): 225-38.

Facorellis Y, Maniatis Y. 1999. Possibilities and accuracy of radiocarbon dating in the Palaeolithic period. British School at Athens Studies 3:179-89.

Gallis K. 1996. The Neolithic world, Neolithic civilisation in Greece. In: Papathanasopoulos GA, editor. N.P. Athens: Goulandris Institution, Museum of Cycladic Art. p 23-37.

Hauptmann H. 1981. Otzaki-Magula III. Das späte Neolithikum und das Chalkolithikum. BAM 21.

Hedges REM, Law IA. 1989. The radiocarbon dating of bone. Applied Geochemistry 4:249-53.

Johansen OS, Gulliksen S, Nydal R. 1986. $\delta^{13} \mathrm{C}$ and diet: analysis of Norwegian human skeletons. Radiocarbon 28(2A):754-61.
Karkanas P. 1999. Lithostratigraphy and micromorphology of Theopetra cave deposits, Thessaly, Greece: some preliminary results. British School at Athens Studies 3:240-51.

Karkanas P, Kyparissi-Apostolika N, Bar-Yosef O, Weiner S. 1999. Mineral assemblages in Theopetra, Greece: a framework for understanding diagenesis in a prehistoric cave. Journal of Archaeological Science 26:1171-80.

Karkanas P, Weiner S. 2000. Lithostratigraphy and diagenesis of the deposits of the Theopetra cave, Kalambaka. In: Kyparissi-Apostolika N, editor. Proceedings of the International Scientific Conference "Theopetra cave-Twelve years of Excavations and Research". Trikala, 7-8 November 1998. p 37-52.

Kitagawa H, van der Plicht J. 1998. Atmospheric radiocarbon calibration to 45,000 yr BP: Late Glacial fluctuations and cosmogenic isotope production. Science 279:1187-90.

Kyparissi-Apostolika N. 1990. Prehistoric inhabitation in Theopetra cave, Thessaly. In: Gallis K, Darmezin L, Decourt JC, Deriziotis L, Doulgeri-Intzessiloglou A, Helly B, Vongraeve V, Intzessiloglou H, Loucas Z, Pantos P, Di Salvatore M, Tzafalias A, Walters Ch, editors. Actes du Colloque International: Quinze années de recherches archéologiques, 1975-1990. Bilans et Perspectives. Lyon. 17-22 Avril 1990. p 103-8.

Kyparissi-Apostolika N. 1999. The Neolithic use of Theopetra cave in Thessaly, Greece. In: Halstead P, editor. Neolithic society in Greece. Sheffield Studies in Aegean Archaeology. Sheffield Academic Press. Volume 2. p 142-52.

Kyparissi-Apostolika N. 2000a. The Neolithic period in Theopetra cave. In: Kyparissi-Apostolika N, editor. Proceedings of the International Scientific Conference "Theopetra cave-Twelve years of Excavations and Research.” Trikala, 7-8 November 1998. p 181-234.

Kyparissi-Apostolika N. 2000b. The excavations in Theopetra cave 1987-1998. In: Kyparissi-Apostolika N, editor. Proceedings of the International Scientific Conference "Theopetra cave-Twelve years of Excavations and Research". Trikala, 7-8 November 1998. p 17-36.

Laj C, Mazaud A, Duplessy JC. 1996. Geomagnetic intensity and ${ }^{14} \mathrm{C}$ abundance in the atmosphere and ocean during the past $50 \mathrm{kyr}$. Geophysical Research Letters 23(16):2045-8. 
Longin R. 1971. New method of collagen extraction for radiocarbon dating. Nature 230:241-2.

Maniatis Y, Kromer B. 1990. Radiocarbon dating of the Neolithic early Bronze Age site of Mandalo, W. Macedonia. Radiocarbon 32(2):149-53.

Milojcic V1J, Boessneck D, Schneider H. 1965. Paleolithikum um Larissa in Thessalien. Bonn.

Mook WG, Streurman HJ. 1983. Physical and chemical aspects of radiocarbon dating. PACT 8(II.1):31-55.

Mook WG, Waterbolk HT. 1985. Sample material and contamination, radiocarbon dating. Handbook for Archaeologists 3:27-31.

Nelson E. 1996. A report on the AMS radiocarbon dating of a human bone from Theopetra cave, Thessaly. Archaeology Department, Simon Fraser University, Burnaby, BC Canada. p 1-10.

Olsson IU. 1979. The importance of the pre-treatment of wood and charcoal samples in Radiocarbon dating. In: Berger R, Suess HE, editors. Proceedings of the 9th International Radiocarbon Conference. Berkeley/Los Angeles: University California Press. p 135-46.

Panagopoulou E. 1999. The Theopetra Middle Palaeolithic assemblages: their relevance to the Middle Palaeolithic of Greece and adjacent areas. British School at Athens Studies 3:252-65.

Perlès C. 1988. New ways with an old problem. In: French EB, Wardle KA, editors. Proceedings of the Congress of Manchester "Problems in Greek Prehistory". Bristol. p 477-88.

Polach HA. 1976. Radiocarbon dating as a research tool in archaeology-Hopes and limitations. In: Barnard N, editor. Proceedings of the Symposium on scientific methods of research in the study of ancient Chinese Bronzes and South East metal and other archaeological artifacts. Australian National University, Department of Far Eastern History Publications.

Runnels C. 1988. A prehistoric survey of Thessaly: new light on the Greek Middle Palaeolithic. Journal of Field Archaeology 15:277-90.

Runnels C. 1993. The Lower and Middle Palaeolithic of Thessaly, Greece. Journal of Field Archaeology 20: 299-317.

Stravopodi E, Manolis S, Kyparissi-Apostolika N. 1999. Palaeoanthropological findings from Theopetra cave in Thessaly: a preliminary report. British School at Athens Studies 3:271-81.

Stuiver M, Polach HA. 1977. Discussion: reporting of ${ }^{14} \mathrm{C}$ data. Radiocarbon 19(3):355-63.

Stuiver M, Reimer PJ. 1993. Extended ${ }^{14} \mathrm{C}$ data base and revised Calib $3.0{ }^{14} \mathrm{C}$ age calibration program. Radiocarbon 35(1):215-30.

Stuiver M, Reimer PJ, Bard E, Beck JW, Burr GS, Hughen KA, Kromer B, McCormac G, van der Plicht J, Spurk M. 1998. INTCAL98 radiocarbon age calibration, 24,000-0 cal BP. Radiocarbon 40(3):112751.

Theocharis D. 1970. History of the Greek nation. Athens: Ekdotiki Athinon. Section A. p 37.

Theocharis D. 1973. Neolithic Greece. Athens: National bank of Greece.

Tsountas Ch. 1908. The prehistoric acropolis of Dimini and Sesklo. Athens.

Voelker AHL, Sarnthein M, Grootes PM, Erlenkeuser H, Laj C, Mazaud A, Nadeau MJ, Schleicher M. 1998. Correlation of marine ${ }^{14} \mathrm{C}$ ages from the Nordic seas with the GISP2 isotope record: implications for ${ }^{14} \mathrm{C}$ calibration beyond 25 ka BP. Radiocarbon 40(1):51734.

de Vries H, Barendsen GW. 1953. Radiocarbon dating by a proportional counter filled with carbon dioxide. Physica 19:987-1003.

Wace AJB, Thompson MS. 1912. Prehistoric Thessaly. Cambridge: Cambridge University Press. 